\title{
WestVirginiaUniversity
}

THE RESEARCH REPOSITORY @ WVU

Graduate Theses, Dissertations, and Problem Reports

2007

\section{Multiagent approach for power system reconfiguration}

\author{
Pinak Tulpule \\ West Virginia University
}

Follow this and additional works at: https://researchrepository.wvu.edu/etd

\section{Recommended Citation}

Tulpule, Pinak, "Multiagent approach for power system reconfiguration" (2007). Graduate Theses, Dissertations, and Problem Reports. 4344.

https://researchrepository.wvu.edu/etd/4344

This Thesis is protected by copyright and/or related rights. It has been brought to you by the The Research Repository @ WVU with permission from the rights-holder(s). You are free to use this Thesis in any way that is permitted by the copyright and related rights legislation that applies to your use. For other uses you must obtain permission from the rights-holder(s) directly, unless additional rights are indicated by a Creative Commons license in the record and/ or on the work itself. This Thesis has been accepted for inclusion in WVU Graduate Theses, Dissertations, and Problem Reports collection by an authorized administrator of The Research Repository @ WVU. For more information, please contact researchrepository@mail.wvu.edu. 


\title{
Multiagent Approach for Power System Reconfiguration
}

\author{
by \\ Pinak Tulpule \\ Thesis submitted to the \\ College of Engineering and Mineral Resources \\ at West Virginia University \\ in partial fulfillment of the requirements \\ for the degree of \\ Master of Science \\ in \\ Electrical Engineering \\ Research Assistant Professor Karl Schoder, Ph.D. \\ Professor Powsiri Klinkhachorn, Ph.D. \\ Professor Ali Feliachi, Ph.D., Chair \\ Lane Department of Computer Science and Electrical Engineering \\ Morgantown, West Virginia \\ 2007
}

Keywords: multiagent systems, power system reconfiguration, termination detection, shipboard power system, agent communication, controller area network.

Copyright 2007 Pinak Tulpule 


\author{
Abstract \\ Multiagent Approach for Power System Reconfiguration \\ by \\ Pinak Tulpule \\ Master of Science in Electrical Engineering \\ West Virginia University \\ Professor Ali Feliachi, Ph.D., Chair
}

The objective of this thesis is to develop an agent based reconfiguration algorithm for power system application with termination detection and to provide communication architecture for these agents. The underlying maximum flow algorithm based on graph theory has proven the concept of autonomous distributed reconfiguration using multiagent systems. The new rule-based algorithm takes into account the requirement of bidirectional power flows, and special power routing for DC devices. Even though the algorithm is self-stabilizing and agents become idle after finite time, implementation of the solution within the actual power system requires that all agents be in an idle state. The process of detecting the idle state of all agents in a distributed environment is called termination detection. After surveying various methods for termination detection, a modified method that is suitable for reconfiguration algorithm is developed. The method uses wave algorithm with tree topology and provides the ability of detecting termination to each of the agents. These reconfiguration agents can perform satisfactorily only if they can communicate and share information with other agents in the system. Thus, it is extremely important to have a uniform agent communication language and messaging scheme among all the agents. With the Controller Area Network as hardware backbone for communication, a new message structure and communication protocol is developed based on CANOpen and standards specified by Foundation for Intelligent Physical Agents. The communication architecture provides easy interface between reconfiguration agents and other agent based systems. The designed algorithms are applied to the reconfiguration of power system on all electric ships. The system is simulated on multiple computers and includes a command and control center to monitor and supervise the entire operation of the system. The integrated system is tested for specific scenarios and statistics are recorded to analyze the algorithm performance and robustness. The evaluation of the system for correctness of reconfiguration solutions and the time required to complete the reconfiguration show promising results. 


\section{Acknowledgement}

I would like to thank my advisor, Dr. Ali Feliachi, for his guidance and encouragement throughout my studies and research work at West Virginia University. I like to express my gratitude to Dr. Karl Schoder for his directions and help during my research work. I am thankful to Dr. Powsiri Klinkhachorn for his support during my studies. I also express my thanks to all the faculty members and students of APERC for their valuable feedback.

I like to thank CSEE department and university for all the facilities and equipment.

I am also thankful to my parents for their love, encouragement and support.

This research is sponsored in part by a grant from the US DEPSCoR and ONR (DOD/ONR N000 14-031-0660). 
Think Globally, Act Locally.

- David Brower (1969) 


\section{Contents}

$\begin{array}{ll}\text { Abstract } & \text { ii }\end{array}$

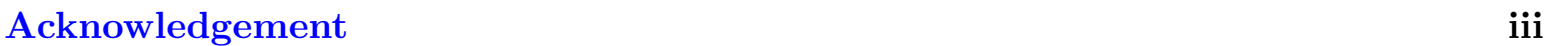

List of Figures $\quad$ viii

List of Tables $\quad x$

1 Introduction $\quad 1$

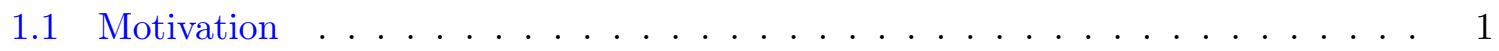

1.2 Problem statement . . . . . . . . . . . . . . . 3

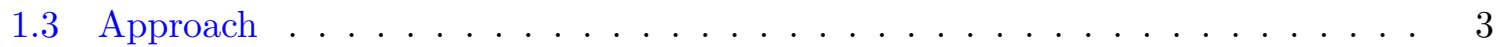

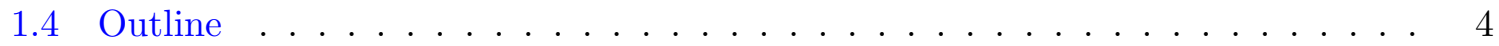

2 Literature Survey $\quad 6$

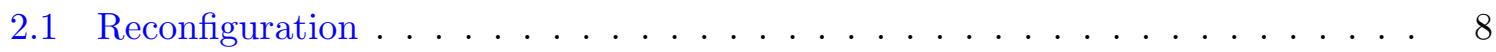

2.2 Algorithm Termination . . . . . . . . . . . . . . . . . 10

2.2.1 Dijikstra-Scholten Algorithm . . . . . . . . . . . . . . . . 12

2.2 .2 Snapshot Algorithms . . . . . . . . . . . . . . . . . . . . . . 12

2.2 .3 Wave Algorithms . . . . . . . . . . . . . . . . . . . 13

2.3 Communication . . . . . . . . . . . . . . . . . . 14

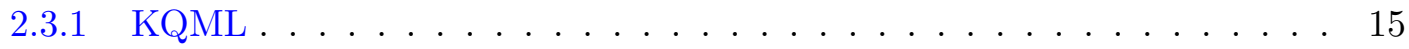

2.3.2 Foundation for Intelligent Physical Agents . . . . . . . . . . . . . 16

2.3.3 FIPA Agent Communication Language . . . . . . . . . . . . . . . . . . 17 
2.4 Controller Area Network . . . . . . . . . . . . . . . . . . . . . . . 18

2.5 Conclusion . . . . . . . . . . . . . . . . . . . 19

3 Reconfiguration Algorithm 20

3.1 Shipboard power system . . . . . . . . . . . . . . . . . 20

3.2 Graph representation . . . . . . . . . . . . . . . . . . . 22

3.2 .1 Graph theory . . . . . . . . . . . . . . . . . 22

3.2.2 Shipboard power system graph . . . . . . . . . . . . . . . . . 24

3.3 Reconfiguration algorithm . . . . . . . . . . . . . . . . 25

3.4 Discussion . . . . . . . . . . . . . . . . . . . . . 31

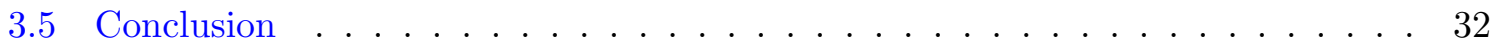

4 Termination Detection 33

4.1 Centralized Approach . . . . . . . . . . . . . . . . . . 34

4.2 Distributed Approach . . . . . . . . . . . . . . . . . . . 35

4.3 Proposed Algorithm . . . . . . . . . . . . . . . . . . 36

4.3 .1 Spanning Forest . . . . . . . . . . . . . . . . 37

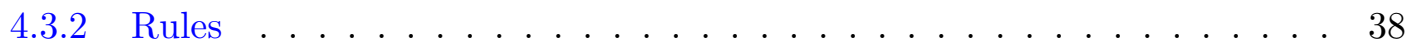

4.4 Discussion . . . . . . . . . . . . . . . . . . . 40

5 Communication $\quad 42$

5.1 System Architecture . . . . . . . . . . . . . . . . . . . . . . 43

5.2 Message Structure . . . . . . . . . . . . . . . . . . . 45

5.3 Message Definitions . . . . . . . . . . . . . . . . . . . 48

5.4 Addressing Scheme . . . . . . . . . . . . . . . . . . 52

5.5 Message Priorities . . . . . . . . . . . . . . . . . 54

5.6 State Diagram Description . . . . . . . . . . . . . . . . . 54

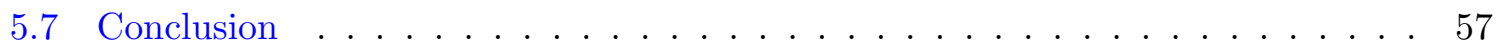

6 System Integration $\quad 59$

6.1 Power system model . . . . . . . . . . . . . . . . . . . . . 61 
6.2 Implementaton agent . . . . . . . . . . . . . . . . . . . . . . 62

6.3 Reconfiguration agents . . . . . . . . . . . . . . . . . 64

6.4 Command and Control Center . . . . . . . . . . . . . . . . . . 64

7 Testing and results $\quad 66$

7.1 Initialization . . . . . . . . . . . . . . . 66

7.2 System startup at full load . . . . . . . . . . . . . . . . 67

7.3 Other test cases . . . . . . . . . . . . . . . . . 70

8 Conclusion $\quad 76$

8.1 Future work . . . . . . . . . . . . . . . . . . 77

$\begin{array}{ll}\text { A Error Codes } & 79\end{array}$

$\begin{array}{ll}\text { References } & 81\end{array}$ 


\section{List of Figures}

3.1 Shipboard power system . . . . . . . . . . . . . . . . . . . 21

3.2 Example of graph . . . . . . . . . . . . . . . . . . . . . . 23

3.3 Graph representation of shipboard power system . . . . . . . . . . . . . 24

3.4 Example of residue assignment f . . . . . . . . . . . . . . . . 28

4.1 Blackboard concept . . . . . . . . . . . . . . . . . 34

4.2 Concept of distributed termination detection . . . . . . . . . . . . . 35

4.3 Example tree and parent and children list . . . . . . . . . . . . . . 37

4.4 Graphical representation of rules one, two and nine. . . . . . . . . . . . . 40

4.5 State diagram, where the Black and White waves denote sending events. . . . . 41

5.1 Device architecture . . . . . . . . . . . . . . . . . . 43

5.2 Message structure example . . . . . . . . . . . . . . . . . . 49

5.3 Communication and device state diagram . . . . . . . . . . . . . 55

5.4 Messages exchanged during device start up . . . . . . . . . . . . . 58

6.1 Integrated system . . . . . . . . . . . . . . . . . 60

6.2 Layered architecture . . . . . . . . . . . . . . . . . . . . 62

6.3 Power system model . . . . . . . . . . . . . . . . . . . 63

6.4 Command and control center - Graphical user interface . . . . . . . . . . 65

7.1 Spanning forest . . . . . . . . . . . . . . . . . 67

7.2 One possible solution to reconfiguration problem for startup case. . . . . . . . 68

7.3 Timing analysis for startup case . . . . . . . . . . . . . . 68 
7.4 Messages exchanged among agents . . . . . . . . . . . . . . . . 69

7.5 Trees and power flow solution for case: line $2-7$ is disconnected. . . . . . . . . . 70

7.6 Test results for case: line $2-7$ is disconnected. . . . . . . . . . . . . . . . 71

7.7 Reconfiguration solution for case: line 2-7 is disconnected and capacity of line

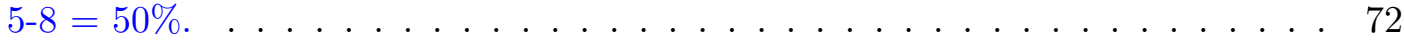

7.8 Test results for case: line 2-7 is restored to full capacity and capacity of line

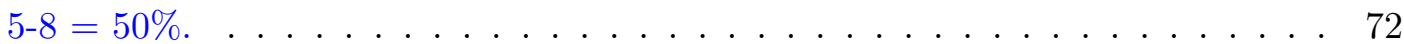

7.9 Test results for case: line 2-7 is disconnected and capacity of line $5-8=50 \%$. $\quad 73$ 7.10 Execution Time . . . . . . . . . . . . . . . . . . . . . 74

7.11 Messages exchanged. CM: Control messages, RM: Reconfiguration Messages. . 74 


\section{List of Tables}

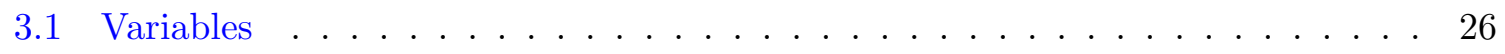

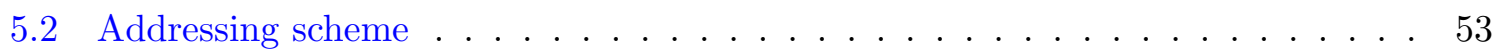

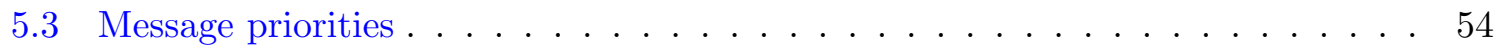




\section{Chapter 1}

\section{Introduction}

\subsection{Motivation}

The past four decades have witnessed an exponential increase in the number and sophistication of electronic systems in many fields of applications ranging from domestic products, life saving apparatus, power systems to automation and control. Semiconductor devices, digital electronics and electronic communication systems have created innovative ways for overall system management. The emerging presence of computers has brought greater efficiency and operational potential in electrical systems. Computers allow performing complex computations providing feasibility of implementing advanced control algorithms. These electronic devices along with computer interface provide advantages of precise control, accurate measurements, flexibility, and intelligence to automatic system operations. Looking at the advantages and reliability of electronic components, more electronic devices are replacing hydraulic and mechanical components. This trend is also visible in electrical power systems with use of electronic actuators and sophisticated power electronics devices to increase the reliability of power generation and distribution network. The spread of electricity generation and distribution system bring up complex problems like power system reconfiguration, automatic 
generation scheduling, load shedding, etc.

Autonomous agents and multiagent systems embody a novel paradigm for analyzing, designing, and implementing solutions to complex problems. Multiagent systems received greater attention in the recent past in many intelligent, distributed systems to model the theories of interactivity in human societies. Humans interact in various ways and at different levels. For instance, humans observe and model one another, they request and provide information, they negotiate and discuss, they develop shared views of their environment, they detect and resolve conflicts, and they form organizational structures such as teams, committees, and economies. These multiagent systems provide a new approach to tackle the problems in power systems. The agent based systems are scalable and capable of working in large geographical areas with reliable communication channels. Multiagent systems provide a promising solution to power system reconfiguration and aid in maintaining the survivability of the system.

Small power systems onboard ships are small when compared to terrestrial power systems due to limited space. In an all-electric ship, the electric power system is important for the entire operation of the system and therefore a vital component of today's ships. The Naval Combat Survivability generation, propulsion, and DC power distribution test beds have proven that incorporating the latest technology will simplify ship design and maintenance. The success of increasingly all electric naval warships requires new approaches to their design and operation. To optimize survivability, lifetime and cost performance, dependable automation strategies, nonlinear control theory, applied analytic redundancy, and distributed intelligence are envisioned to form robust networks and allow reconfiguration based on situational awareness. The realization of these goals is hindered by several technological shortcomings including incomplete theoretical basis and limited experience in autonomous distributed control. Multiagent systems could be successful in improving the autonomy and survivability of power system reconfiguration. 


\subsection{Problem statement}

The goal of this thesis is to design an agent based energy management system for electric shipboard power systems. This system is a combination of AC and DC distribution systems and therefore provides a challenge for power system reconfiguration. The goal of the algorithm is to reconfigure the power system within finite time and supply all the requested power demand considering load priorities. The objectives for the multiagent reconfiguration system are:

- All load demands should be satisfied.

- In case of reduced power generation or system constraints, all higher priority loads should receive the demanded power first.

- The reconfiguration solution should complete in finite time.

- All agents should simultaneously discover the end of the reconfiguration algorithm.

- Every agent should send appropriate control signals to the corresponding power system component after successful termination.

- The reconfiguration system should work without any central controller or central database (blackboard).

\subsection{Approach}

The electric shipboard system is modeled using graph theory with links representing the power lines and nodes representing the power system components. Each node acts as an agent and executes the reconfiguration algorithm independently. The reconfiguration algorithm is based on a maximum flow algorithm. Since the agents execute independently and interact only with their neighbors, another algorithm is necessary to find the consistent state of all the agents 
and determine whether all agents have finished the reconfiguration. This algorithm is called "termination detection" algorithm.

The agents need a communication channel to interact and share information; therefore they require a reliable, error free communication channel. Controller Area Network bus is chosen as the communication medium with FIPA (Foundation for Intelligent Physical Agents) agent communication language for streamlined communication among agents.

\section{Assumptions}

- The power system and communication hardware controllers are capable of detecting fault in the system.

- Protection devices act first, followed by reconfiguration agents to restore and reconfigure the system.

- Communication links are ideal, there is no traitor agent in the system, and agents execute algorithms without any error.

\subsection{Outline}

An outline of the remaining thesis is as follows:

Chapter 2 presents a survey of multiagent systems, different reconfiguration algorithms proposed for power system applications, a termination detection algorithm, and communication protocols available for agent based systems.

Chapter 3 develops the proposed agent based reconfiguration algorithm. First two sections explain power system modeling in graph theory and last two sections present the reconfiguration algorithm.

Chapter 4 describes the termination detection algorithm used with reconfiguration algorithm. 
Chapter 5 presents message structure, priorities, agent database and other methods for agent communication and system maintenance.

Chapter 6 gives the overall system architecture with the tasks and operations of command and control center.

Chapter 7 presents selected test cases, distributed test setup, and results. Conclusion and possible future work are given in Chapter 8. 


\section{Chapter 2}

\section{Literature Survey}

In the past ten years a transformation in the field of agent based software design has taken place: the term "agent" evolved from an artificial intelligence community buzzword, often accompanied by overly optimistic promises of amazing feats the agent will be capable of, to a valid paradigm for software development rather than a class of artifacts. This paradigm emphasizes autonomy, both at design time and runtime, adaptability, and cooperation. The agent paradigm seems appealing in a world of networked, distributed and heterogeneous systems [47]. There is no consensus over the definition of agents but a possible definition appropriate to this thesis work is as follows - an agent is an autonomous computational entity such as a software program that can be viewed as perceiving its environment through sensors and acting upon this environment through its effectors [74]. An agent possesses its own resources that allow a partial representation of its environment, communicates directly to offer some services, and responds to requests from other agents $[34,71]$. Software agents are capable of flexible autonomous actions [73] characterized by situational awareness, reactivity, pro-activeness, and social ability. A description of agent's properties is as follows,

- Autonomy is the agent's ability of either acting without human intervention [35] or of learning from experiences [52]. 
- Reactivity means that an agent should be able to respond in a timely manner to any changes in its environment.

- Proactiveness is the ability of an agent to take initiative and be opportunistic in its behavior.

- The social ability is not limited to communication and data transfer only, but rather it refers to the ability of an agent to negotiate a task or cooperate with other agents to complete the global objectives of the system [75].

- The situational awareness is the ability of agents to perceive changing system conditions and take decisions to satisfy the need of the overall system [73].

Distributed systems started in the 1980s with the advancement of distributed computing, i.e., using multiple processors for complex computations. Distributed systems can be homogeneous or heterogeneous. All agents in a homogeneous system perform the same tasks and the performance is increased by parallel processing of data. In heterogeneous systems, different types of agents perform different tasks and cooperate to satisfy the requirements of the entire system. The need for distributed systems led to the development of different algorithms, system architectures and communication infrastructures. KQML (Knowledge Query and Manipulation Language) is one of the communication languages developed as an effort to facilitate communication between heterogeneous controllers [22]. Developments in industrial automation also required new communication networks that led to development of fieldbus technology. CAN bus is a fieldbus, originally designed for automotive use, and extremely useful in harsh and noisy environments with short distance communication requirements $[48,8]$.

The remaining sections of this chapter give a survey of agent applications in power systems, reconfiguration or restoration algorithms, distributed termination algorithms and their applications in reconfiguration system, and languages and communication protocols for agent based communication. 


\section{$2.1 \quad$ Reconfiguration}

Several researchers have addressed distributed and centralized reconfiguration strategies for power systems $[65,33,32,67]$. These papers provide background information on different strategies for control and reconfiguration of shipboard power systems. The paper by Solanki gives a brief overview of work done at Mississippi State University [65] and discusses the MATLAB and JADE implementations for simulating agent based systems. Huang and Cartes [32] used agents for power flow control in systems with ring and mesh topology using contract based negotiations. The agents use input-output cost curves and then assign power to different loads. Their work includes a spanning tree to prevent redundant information accumulation due to loops in information flow. In the thesis [33] by Huang, a fast reconfiguration strategy is developed by representing the shipboard power system as graph matrices, using negative power balance to decide on load shedding, and fast search methods to identify possible loads to shed. Sarma and Butler-Purry [10] considered a probability based predictive reconfiguration strategy, where the algorithm analyses possible threats to the power system before a missile hit and isolates the area of potential damage. A rule based expert system solution is provided for shipboard power system restoration in [67]. The expert system analyses the affected area and restores power to the loads according to their priority.

Several efforts on applying the agent technology to AC power distribution systems [50] for system reconfiguration, market simulation, pricing, etc, have been undertaken. Zhang et al. [77] present a review of multiagent systems, distributed computing, communication and data integration needs in the power industry with an example on condition monitoring and maintenance system. The example system is a heterogeneous multiagent system, i.e., the system contains different types of agents performing different tasks. The system contains diagnostic agents to provide system diagnostics, an alarm agent that reports any fault in the system, maintenance agent decides maintenance tasks by analyzing the reports from diagnostic agents. In addition, the maintenance agents at different utilities coordinate with each other to develop 
a schedule for system maintenance. In [36] authors proposed a feeder reconfiguration method to minimize the total cost that is the summation of reliability, feeder resistive loss, investment, and maintenance costs. They formulated the problem as a non-linear, constrained, non-differentiable optimization problem and solved it using simulated annealing, which is a combinatorial optimization algorithm, to obtain a globally optimal solution. Another work by Arias-Albornoz and Sanhueza-Hardy [6] suggests a feeder reconfiguration strategy aiming at reducing the total energy buying costs in a distribution network. There is a commercial patented product by S and C Electric Company called IntelliTEAM II ${ }^{\mathrm{TM}}$ Automatic Restoration System [68, 2]. The system comprises of virtual agents with peer-to-peer communication working in teams and a mobile team agent to coordinate their decisions. The agents react to faults and work in teams to restore power to the affected area without any source or line overloading according to load priorities. The agents use voltage and current data gathered prior to the fault to develop a restoration method for the affected area.

The above research addresses agent based reconfiguration for power flow considering appropriately controlled dynamic system. Authors in [70] used nonlinear methods, like Lyapunov and Polytopic analysis to characterize the stability characteristics of systems with negative resistance of power converters and performed time domain simulations of the zonal DC distribution systems. Their work compared methods for stability analysis and presented a method to determine regions of asymptotic stability. Authors from the University of Texas developed control strategies to rapidly assess the stability of a control action, and to find a sequence of discrete control steps that steer the system to a target equilibrium state in a safe and stable fashion [12]. Use of Lyapunov energy functions to find the region of stability for each configuration avoids the necessity of individual trajectory simulations. The discrete events occurring during reconfiguration can also lead to angle instability; however, since the synchronism between generators in tightly coupled electric shipboard power system is strong, in part due to the parallel operation of the generators, angle instability is not a critical problem [49].

Previous research at West Virginia University used multiagent systems to reconfigure the 
shipboard power system. In the thesis by Shilpa Ganesh [27], graph theory and maximum flow algorithm are used to solve the power flow problem. Power system is modeled by representing power lines by links and each device or connection by a node. Each node acts as an agent and communicates only to the agents directly connected by links. The reconfiguration problem was formulated as maximum flow problem and rule-based system was designed to solve this problem.

The distributed reconfiguration system presented in [27] is based on a blackboard architecture, i.e., a central memory is used for data sharing between all the agents in a system. This central memory hampers the main definition of distributed agent based systems as all agents depend on this common memory for their operation. The blackboard was necessary for two reasons. First, to detect the existence of a consistent solution for the reconfiguration problem in the distributed system, and second, to simulate the communication between agents. Therefore, to develop a true distributed system it is necessary to find an alternative approach to both these situations. Distributed termination detection algorithm and communication structure are proposed here to solve these problems.

\section{$2.2 \quad$ Algorithm Termination}

Termination detection $[46,40]$ and consensus problem $[51,9,39]$ are two similar problems in distributed computing for drawing common conclusions based on individual process decisions. Some other widely discussed problems are agent coordination and distributed planning $[18,41]$ in heterogeneous multiagent systems.

Consensus is a special problem in distributed computing where all processes start with an initial value and are supposed to eventually output a value of the same type. The condition is that the output values of all processes must be the same, i.e., all agents should agree. All processes should converge on the same value. One classic consensus problem is the coordinated attack problem where several generals are planning a coordinated attack on a common 
enemy from different directions, and the only way the attack can succeed is if all generals attack simultaneously. The restriction is that only nearby generals can communicate with an unreliable communication channel (i.e., a messenger) [39]. So the generals have to come up with a common belief of whether to attack or not, i.e., all generals should have a consensus on one answer.

Consensus is generally simple to achieve with an ideal communication channel and simple process dynamics. Work by Bracha and Toueg gives a protocol for reaching agreement in case of faulty processes [9]. They considered two faults; fail-stop processes and malicious processes. A survey of consensus methods given in [51] introduces algorithms for multiagent coordination with time-invariant and dynamically changing information exchange. Use of directed graphs and nonnegative matrix theory to represent the consensus problem is very common. [43, 42] present an approach based on these concepts and give a method for determining consensus for simple integrator agents.

Termination detection algorithms have a long history in the field of distributed computing and concurrent programming [23]. In termination detection, the goal is to find the state of all agents in the system and to determine whether all agents are idle. Termination is a global property and difficult to detect as each process has knowledge of only its local state and the states of processes connected through direct communication channels. References [23, 39, 72] give an extensive survey and comparison of various termination detection algorithms. One set of termination detection algorithms requires a unique node to start computations and detect termination. Two examples for this type of algorithm, which are known as diffusing algorithms, are the Dijikstra-Scholten algorithm [39] and credit recovery algorithm [72]. Other algorithms are trying to take a consistent snapshot of the current computation to determine its status. Examples include the Snapshot algorithms [39] and Chandy-Lamport Algorithm [39]. Another class of algorithms allows any node to start the detection algorithm. Examples are wave based algorithms [72] and graph relabeling algorithms [30]. The following sub-sections give brief overviews of some of these termination detection algorithms. 


\subsubsection{Dijikstra-Scholten Algorithm}

Termination-detection algorithms for asynchronous networks with an underlying graph $\mathrm{G}$ and arbitrary undirected edges are available for diffusing algorithms $A$ [39]. An algorithm $A$ is said to be diffusing when all its activities start at a location where the input occurs and diffuses through some portion of the network via messages. A global state of $A$ is said to be idle when no agent is performing any action and no message is in any communication channel between two agents. The termination-detection problem for $A$ can be summarized as follows:

if, sometime after an input occurs at some agent $i$, algorithm $A$ ever reaches a quiescent global state, then eventually a special done $e_{i}$ output should be performed at node $i$.

The Dijikstra-Scholten algorithm [39] constructs a spanning tree of the agents currently involved in $A$ along which the messages are sent. Furthermore, each agent acknowledges messages in $A$ immediately but the first. The sender of the first message is known as parent node. The tree is allowed to shrink again when two conditions are fulfilled at node $j$ : first, agent $j$ is idle, and second, all its outgoing messages have been acknowledged.

\subsubsection{Snapshot Algorithms}

Another algorithm type belongs to the family of global snapshot algorithms [39]. A snapshot is "consistent" if it looks to the processes as if it was taken at the same instant everywhere in the system. The snapshot algorithms can be used for several purposes including determination of global properties, distributed debugging, and stable property detection. The stable property detection can be used for the special purpose of detecting termination. Two possible approaches are discussed next.

Logical-time Snapshot Algorithm Due to the problems with real time in distributed systems, attempts have been made to create different concepts. One of them is the logical time by Lamport. The idea is to assign to every event of an executing algorithm a "logical 
time," for example, a number from the set of nonnegative integers. These assigned logical times need not relate to real time but need to keep the ordering among interdependent events. Lamport's algorithm maintains local clocks and advances them with every internal (executing a local action or sending a message) and external (receiving a message) event.

Chandy-Lamport Algorithm The Chandy-Lamport algorithm [13] does not use an explicit logical time but rather uses "marker" messages to indicate the point in time that separates states and messages before and after the snapshot. The Chandy-Lamport algorithm augments the original algorithm $A$ and yields the modified implementation $C L(A)$. An agent $i$ may trigger the algorithm itself or be triggered through a "snap" message. After receiving the snap message, the agent records its state and sends "marker" messages to its neighbors. Afterward, it records the messages arriving to keep records of the channels' states until it receives markers for the respective channels. In case an agent receives a marker before it has recorded the state, it records its state, sends out marker messages, and starts recording incoming messages.

Snapshot algorithms can also be used for detecting deadlocks in the reconfiguration algorithm, as deadlock is a special case of a stable global property: a deadlock consists of a cycle of two or more processes, which wait for each other to release resources with no messages in channels connecting them.

\subsubsection{Wave Algorithms}

Wave algorithms [72] are computations eventually performed by every node using local information and information received from other agents. Many times wave algorithms are part of more complex algorithms to achieve the final algorithm. For example, waves may be used to broadcast messages in the distributed system to "wake up" nodes to start processing data. A wave algorithm can be started by a fixed initiator or any arbitrary node in graph $G$. Once 
initiated by a node, the computation propagates throughout the system and returns to the initiator by a message passing scheme. When a node performs wave computations, it is "visited" by the wave. The termination detection algorithm based on waves propagates throughout the system through a variable "wave color." During the computation, each node colors the wave black if the node is active and white if the node has been idle since the last visit of the wave. When the computation reaches the initiator it generates a "decide" event. If the color of the received wave is white then the decision is true otherwise, it is false. If the decision is true then the algorithm detected termination, and if the decision is false then the node restarts the wave computation through another white wave.

\subsection{Communication}

Communication is an inherent property of multiagent systems. All agents need to communicate with their neighbors to achieve their local goals as well as to meet the global objectives. Therefore, the communication interface should be error free, reliable, and the agents should use a common language with a message structure and addressing scheme to exchange messages. The agents are deaf and dumb from the system and are useless if they cannot communicate [69]. Agents need a common language to interact in a shared environment, hiding the details of their internal structure, and build communities of agents to tackle problems that no individual agent can. The concept of agent communication language comes from the evolution of human social abilities and languages. Languages for communicating agents promise to play the role that language played for their human counterparts. Human languages developed as a need for cooperation and coordination to improve the capabilities in a society [76]. Similar need arises when multiple controllers and processes start collaborating intelligently to control an operation, or share and process information with common goals and beliefs. [29] and [54] used agent learning scenarios to show the effectiveness of agent communication language on agent behavior. The authors showed that the evolving communication can be expressed as state 
machine and demonstrated that better communication structure increases the performance of multiagent systems.

Many efforts are in place to enable the social behavior and provide a common language for agent communication. The Knowledge Sharing Effort (KSE) was initiated in 1990 by DARPA and it enjoyed the participation of dozens of researchers from both academia and industry [37]. Its goal was to develop techniques, methodologies, and software tools for knowledge sharing and knowledge reuse, at design, implementation, or execution time. The central concept of the KSE was that knowledge sharing requires communication, which in turn, requires a common language. Therefore, KSE focused on defining the common language. Knowledge Query and Manipulation Language (KQML) is the result of this Knowledge Sharing Effort [38]. The KSE defined ontology and methods to share and process information with streamlined data access. As the agents became more and more intelligent, the operations increasingly became more complex and the communication requirement extended to actions and requests rather than just information querying. Foundation for Intelligent Physical Agents (FIPA) is another such effort for defining agent architecture and communication methods for multiagent systems. Originally formed as a Swiss based organization in 1996 to produce software standards, specifications for heterogeneous and interacting agents and multiagent systems. Now, FIPA is the 11th IEEE Computer Society standards organization that promotes agent based technology and the interoperability of its standards with other technologies [1]. FIPA has specified formal Agent Communication Language (ACL) along with communicative acts, ontology, and semantics that consider the needs of modern agent negotiations.

\subsubsection{KQML}

KQML was conceived as both a message format and a message handling protocol to support run time knowledge sharing among agents. The key features of KQML may be summarized as follows [21] [22]: 
1. KQML messages do not merely communicate sentences in some language, but they rather communicate an attitude about the content (assertion, request, and query).

2. The language's primitives are called performatives. As the term suggests, the concept is related to speech act theory. Performatives define the permissible actions that agents may attempt in communicating with each other.

3. An environment of KQML speaking agents may be enriched with special agents, called facilitators that provide such functions as: association of physical addresses with symbolic names; registration of databases and/or services offered and sought by agents; and communication services (forwarding, brokering, etc.). To use a metaphor, facilitators act as efficient secretaries for the agents in their domain [22].

\subsubsection{Foundation for Intelligent Physical Agents}

Since its beginning, FIPA has played a crucial role in the development of agents' standards and has promoted a number of initiatives and events that contributed to the development and uptake of agent technology [1]. It specifies a reference model for agent platforms [56] that includes Agent Management System (AMS), Directory Facilitator (DF), Agent Communication Channel (ACC), and Message Transport System (MTS). Agent platform (AP) can be thought of as a container or a controller holding a group of agents. AMS exerts supervisory control over the agent platform and helps maintain and record addresses of all agents present in an AP. DF provides yellow page directory service to agents, it keeps a list of different agents in the system, their addresses and the tasks performed by them and finally the MTS performs actual task of sending a message from one agent to other agents. MTS provides the message transport protocol [59] along with message structure using FIPA ACL. 


\subsubsection{FIPA Agent Communication Language}

The FIPA Agent Communication Language (ACL) is a standard message language and sets out the encoding, semantics, and pragmatics of the messages. FIPA ACL specification and representation is similar to KQML. The FIPA ACL has three important and interesting features [7].

1. It is independent of the actual content, since it only defines the communicative act or intention.

2. Its formal semantic element is defined in terms of the feasibility preconditions and the rational effect that allows a communicative act to be scheduled and planned as a normal action (e.g. opening the door); in addition, the semantics allows an agent to consider a message in an explicit manner, if and when needed.

3. The ACL provides the bases for the specification of interaction protocols and common patterns of conversations among agents aimed at specifying high-level tasks, such as delegating a task, negotiating conditions, and some forms of auctions.

ACL is based on a semantic language using prepositions, objects and actions. The semantic language is a formal language and used to define the meaning of the message representing belief, uncertainty, choice (or goal), and action of an agent. FIPA also gives specifications for ACL representation, message headers, transport message envelop and ontology. FIPA ACL is now becoming a standard for agent communication and is used in many agent based applications for maritime systems $[4,19]$. FIPA ACL is considered as formal agent communication language to provide the interoperability among different agent based systems although KQML is still extensively used in distributed process automation $[7,11]$. 


\subsection{Controller Area Network}

Many options for communication architectures exist including Ethernet, wireless, serial bus, field bus technologies, etc. CAN is one field bus technology and originally designed for communication among distributed controllers in automotive industry [8]. Today, CAN networks are used in many real time distributed industrial applications [31]. Such applications cover the drinking or used water adduction; the transportation and the distribution of electricity; purification stations; oil and gas field explorations; the automation and robot systems in mass production; the distributed processing systems, etc. One example is given in [63] where a multiagent system is designed for building automation using field bus technology and agents negotiate by contract and bidding process. There are different types of protocols like DeviceNet, ContractNet and CANOpen. These protocols are applicable for communication based on CAN bus [44, 48]. Contractnet is a protocol that specifies message structure and methods for contract based negotiations among various entities [64] and is widely used as task coordination mechanism [3]. Devicenet is a protocol developed for industrial applications of CAN bus with large number of devices [44] especially where many distinct devices take part in a process operation. CANOpen [14] is an extensive and detailed specification of the OSI application layer for CAN bus. It specifies data structure that is applicable to many different types of controllers and devices. CANOpen is also widely used in industry and well developed for different types of applications [16, 15].

CAN messages are broadcasted over a serial bus with real-time non-destructive message arbitration. CAN nodes use message filters to receive selected messages. There are certain schemes designed for multicast transfer of data like XTP (eXpress Transfer Protocol), VMTP (Versatile Message Transaction Protocol). In MMTP (Multicast Message Transport Protocol) as given in [66], and devices involved in one transfer make a temporary contract and decide on a common address. This address is then used for subsequent transfers until the contract expires. Because of the overhead associated with negotiating a contract this protocol is not 
suitable in applications with smaller controllers. Certain protocols like Contractnet [64] use fixed addressing schemes and each agent receives messages that are directly addressed to it.

\subsection{Conclusion}

This chapter gave an overview of past and current research in distributed computing, agent based systems and agent communication. It gave a theoretical background of different termination detection algorithms. Current power system reconfiguration schemes with specific application to shipboard power systems are summarized. In addition, various communication protocols that are applicable to agent communication are discussed and some examples are given. 


\section{Chapter 3}

\section{Reconfiguration Algorithm}

This chapter develops the proposed agent based reconfiguration algorithm. The electric power system is modeled as directed graph and each node is represented as an agent. The agent monitors links connected to it and node parameters to decide on the reconfiguration strategy. First two sections explain power system modeling in graph theory and last two sections present the reconfiguration algorithm.

\subsection{Shipboard power system}

A schematic of a typical shipboard power system model is shown in Figure 3.1. The system consists of two AC generators, two AC propulsion loads, AC to DC converters, and DC distribution system [45]. The loads are divided into three zones. Zonal distribution reduces the amount of cabling and watertight penetrations required, and simplifies damage control and maintenance. Each zone generally contains one or more load centers that supply power to electric loads in that zone [53]. The overall DC distribution system is divided into two DC buses viz. port bus and starboard bus. These buses can be supplied from any of the main generator units through a Power Supply (PS). Breakers are used to isolate zones from each 


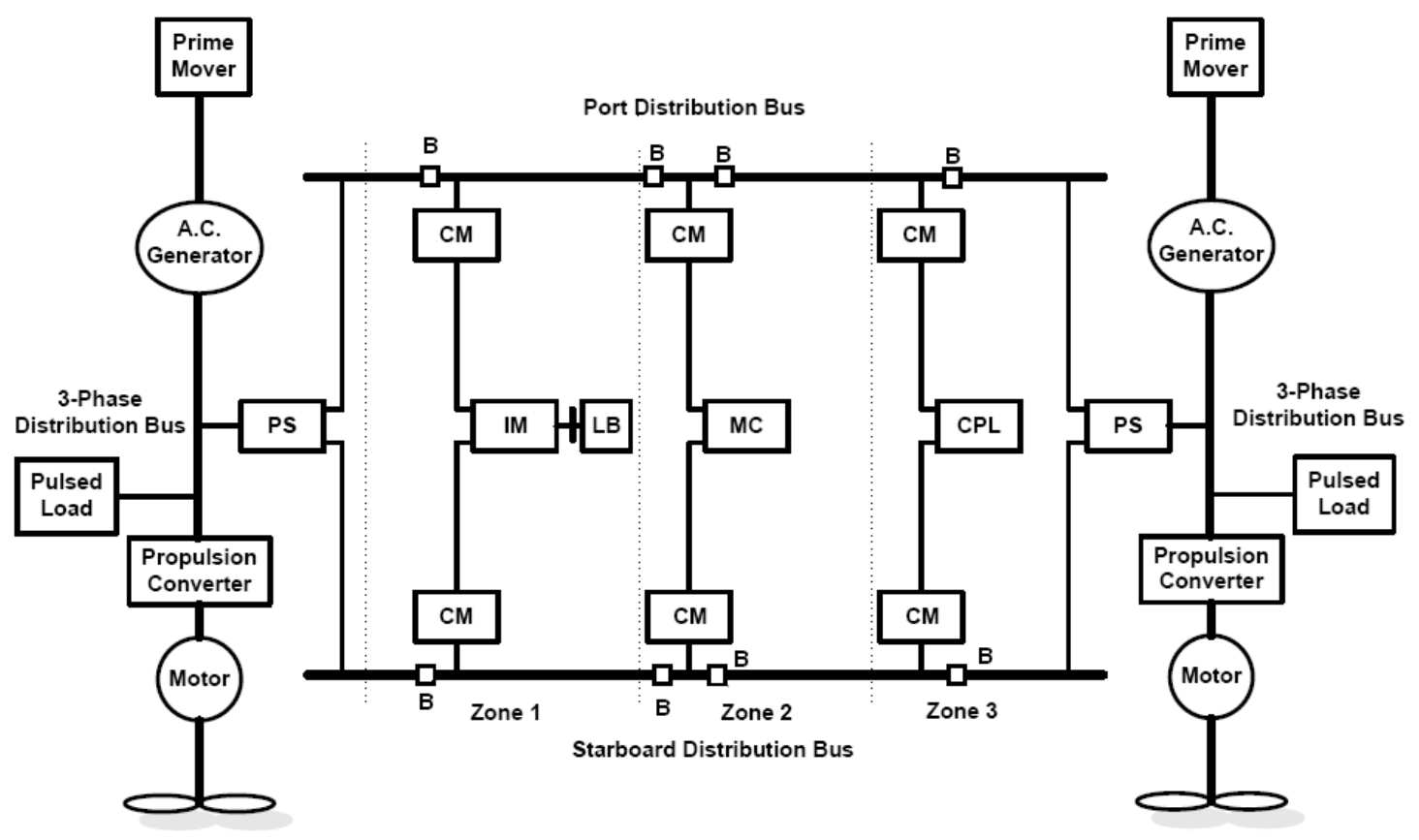

Figure 3.1: Shipboard power system where PS: Power Supply, CM: Converter Module, IM: Inverter Module, LB: Load Bank, MC: Machine Controller, CPL: Constant Power Load, B: Breaker.

other. Each zone may contain an alternate power source and the load centers are capable of supplying normal or alternate power to the loads. Each zone has two converter units, each connected to one bus. These units supply power to zonal loads. All the loads in the system are classified according to their priorities into three groups: vital, semivital, and nonvital. These classes are described as follows: [53]

1. Nonvital (Nonessential) - Readily shedable loads that can be immediately disconnected without adversely affecting ship operations, survivability, or life. Examples are hotel loads such as heating and galley; ship, avionics, and ground support equipment shops; aircraft fueling systems; refrigeration systems; and other loads that can be shut down for a short time until full electric power capability is restored.

2. Semivital (Semiessential) - Loads important to the ship but that can be shut down or 
switched to the alternate plant in order to prevent total loss of ship's electrical power. Examples include aircraft and cargo elevators, deballasting compressors, assault systems, some radar, communications, and seawater service pumps.

3. Vital (Essential) - Nonsheddable loads that affect the survivability of ship or life. Power to these loads is not intentionally interrupted as part of a load-shedding scheme. Examples of vital loads are generators, boilers, and their auxiliaries; close-in weapon systems; electronic countermeasures; tactical data system equipments with volatile memories; medical and dental operating rooms; and primary air search radar.

The above described shipboard power system model is used to study the reconfiguration algorithm. The power system is modeled using graph theory and a rule based system is developed for reconfiguration. Next section introduces graph theory and graph representation.

\subsection{Graph representation}

\subsubsection{Graph theory}

Graph theory is a domain that lies on the cusp of several fields of interest, including applied mathematics, computer science, engineering, management and operations research. The maximum flow problem is an elementary problem in graph theory and combinatorial optimization. The maximum flow problem seeks a feasible solution that sends the maximum amount of flow from a source to a sink node and will be useful in the design of the energy management system. In the graph theory application to power system networks, graph theory can simplify power system representation, make system topology and operations visual, and represent all system topology changes as graph operations.

A graph consists of a set of points and a set of line segments [33]. A graph $G=(V, E)$ consists of two sets $\mathrm{V}$ and $\mathrm{E}$, where $\mathrm{V}$ represents vertices and $\mathrm{E}$ represents edges. If the end points 

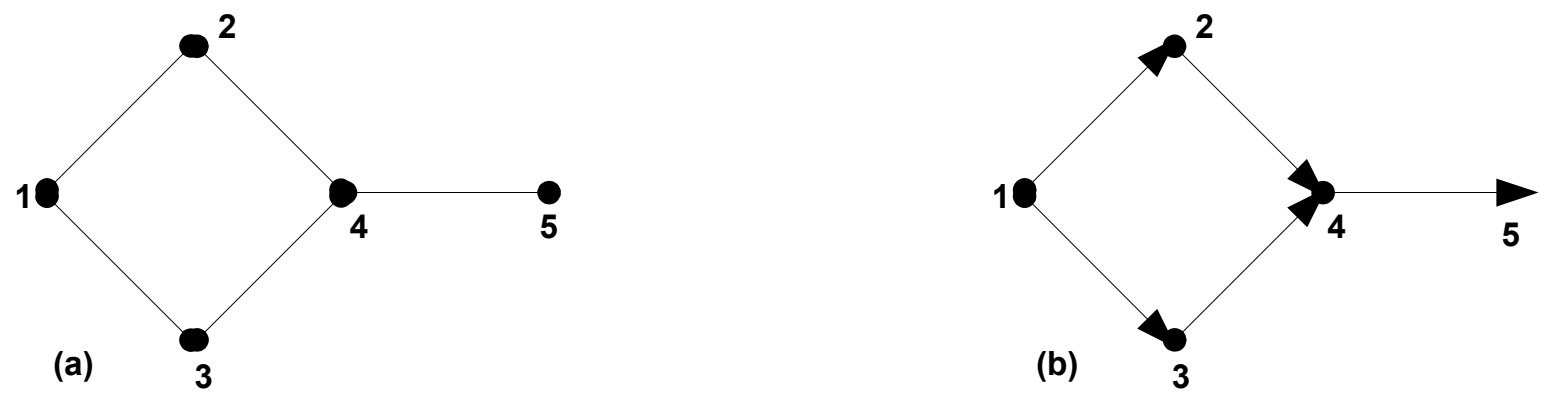

Figure 3.2: Example of graph

of edge $e_{i, j}$ are $v_{i}$ and $v_{j}$, then this edge is represented as $e_{i, j}=\left(v_{i}, v_{j}\right)$. The graph is a directed graph or digraph when the edges have a direction associated with them. For a directed graph, if $e_{i, j} \in E$ then $e_{j, i} \notin E$ for all $e \in E$. Figure 3.2 shows an example of undirected (a) and directed graph (b). For this graph, $V=\{1,2,3,4,5\}$ and $E=\{(1,2),(1,3),(2,4),(3,4),(4,5)\}$. A graph where numerical values are associated with links is called as weighted graph, e.g., if the graph represents a power system then each link can have flow variable associated with it.

The maximum flow algorithms have a variety of important applications [28]. A list includes the Feasible Flow Problem, Problem of Representatives, Matrix Rounding Problem, Scheduling on Uniform Parallel Machines, and Distributed Computing on Two-Processor Computer etc. Many different sequential and parallel algorithms have been developed. All the parallel algorithms but the one by Ghosh, Gupta, and Pemmaraju in [28] present implementations that do not run in polylogarithmic time on a polynomial number of processors. Other advantages of this algorithm are: simple, passive, self-stabilizing, and local checking and corrections only. The algorithm has been applied to find a solution to the power flow problem of the electric shipboard [27]. As the name suggests, the maximum flow algorithm maximizes the flow for given line capacities and load demands. The maximum flow problem arises in a wide variety of situations and in several forms. 


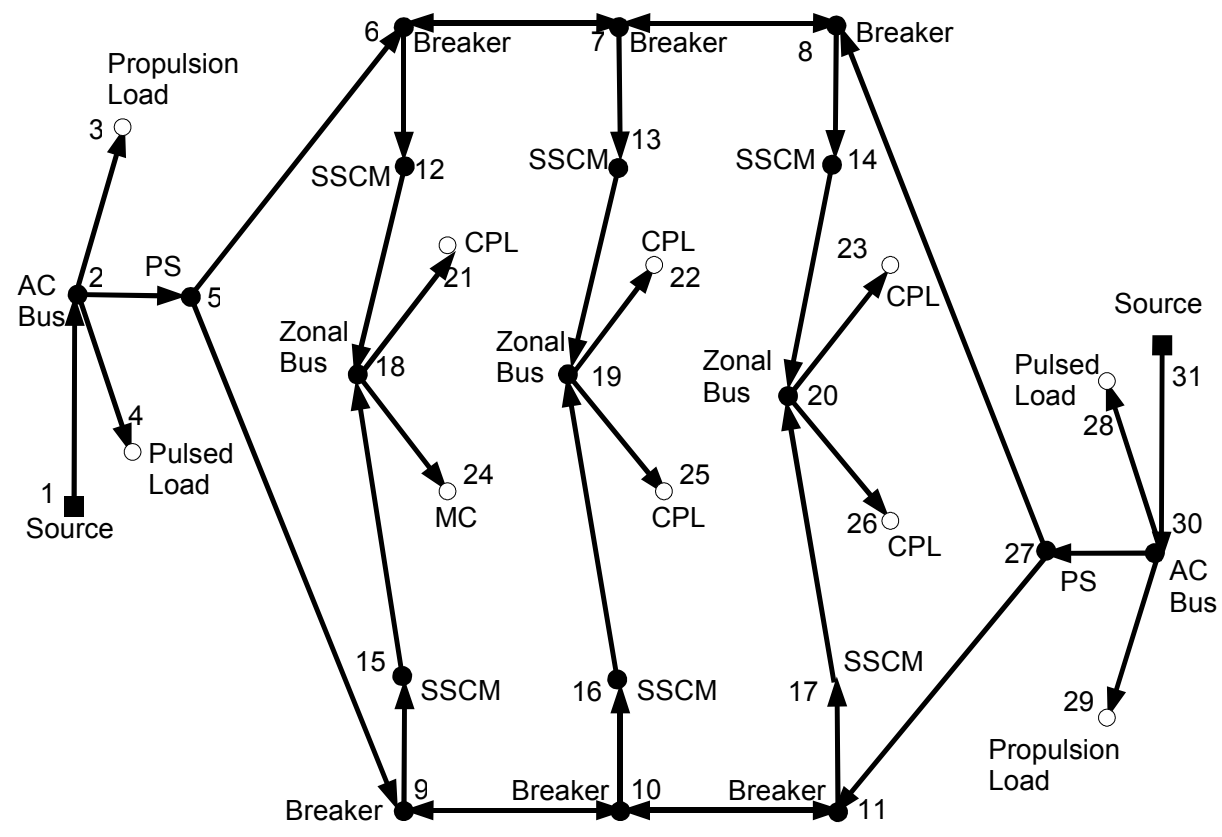

Figure 3.3: Graph representation of shipboard power system

\subsubsection{Shipboard power system graph}

The electric shipboard power system (SPS) is modeled as a directed graph as shown in Figure 3.3. The transformation of the block diagram in Figure 3.1 into the directed graph is achieved by representing physically linked components by neighboring nodes in a graph. Every component that provides control over the power flow is represented as a node. Each of the edges between nodes is associated with a direction because the electric power flows only from the AC generators toward the loads during normal operation. The bidirectional power flow over DC bus is represented by two parallel links with opposite directions. Every load is associated with a priority value that can be vital, semivital or nonvital as specified in Section 3.1. 


\subsection{Reconfiguration algorithm}

Goal of every agent is to satisfy load demand, try to supply requested power to high priority loads, and never exceed the power rating of the link. Each agent monitors the links and local demand and makes decisions to satisfy its goals, i.e., it performs operations like requesting power for a load, rerouting the power flow according to load priorities, etc. The agents' actions are taken without synchronization between two agents. Algorithm presented in [27, 26, 20] provides a framework for SPS energy management system using maximum flow algorithm from $[28]$ and is extended here.

The significant difference from the previous maximum flow algorithm [28] is the graph representation. The system graph contains parallel links with opposite directions (loops of length 2). However, only one link can carry current at any time, if flow $w_{i, j}>0$ then flow $_{j, i}=0$ and vice versa. This constraint is considered in calculating the residue over a link. The reconfiguration algorithm is a set of guarded statements. A guarded statement is a rule in the form of if $<$ condition $>$ then $<$ action $>$. When the guard or condition becomes true, the actions are executed. All actions are assignment statements, i.e., these statements change

values of some variables. For a node $n$ residual graph $G_{f}^{n}$ is formed as, $G_{f}^{n}=\left(V_{f}^{n}, E_{f}^{n}\right)$, Where a node $i \in V_{f}^{n}$ iff $d(i) \geq 0$ and a link $(i, n) \in E_{f}^{n}$ iff $r(i, n)>0$ or $f(i, n)>0$.

\section{Guarded statements}

\section{GS1: Change D-Value}

D-value of an agent is the number of links between the agent and the nearest source agent. Every agent checks if it has unsupplied demand and residual capacity is not available available on any incoming links. If this condition is met then it sets d-value to -1 . Otherwise, it follows the rules mentioned in [27]. D-value $=-1$ indicates that the agent has unsupplied demand that cannot be supplied. This action requests other agents on outgoing links to change their power 
Table 3.1: Variables

$$
\begin{aligned}
& \mathrm{V}=\text { Set of nodes } \quad \mathrm{E}=\text { Set of links } \\
& \mathrm{n}=\text { Agent / node } \quad \ell=\text { link } \\
& \mathrm{d}(\mathrm{n})=\mathrm{d} \text {-value of node } \mathrm{n} \\
& (i, j)=\text { link from } i \text { to } j \quad f(i, j)=\text { Flow on link }(i, j) \\
& c(i, j)=\text { Capacity of link }(i, j) \quad r(i, j)=\text { Residue on link }(i, j) \\
& \mathrm{SD}=\text { Supplied Demand } \quad \mathrm{SDp}=\text { Supplied Demand priority } \\
& \text { UD }=\text { Unsupplied Demand } \quad \text { UDp }=\text { Unsupplied Demand priority } \\
& I=\text { Set of incoming links } \quad I_{a}=\text { Set of active incoming links }(f>0) \\
& O=\text { Set of outgoing links } \quad O_{a}=\text { Set of active outgoing links }(f>0) \\
& f_{I}=\sum f\left(I_{a}\right) \quad f_{O}=\sum f\left(O_{a}\right) \\
& \text { demand }=f_{O}-f_{I}
\end{aligned}
$$

source, if possible, i.e., $\mathrm{d}$-value $=-1$ informs other agents of a bottleneck in the power system so that these agents can act to remove the bottleneck by looking for an alternate source.

Listing 3.1: GS1 Update d-value

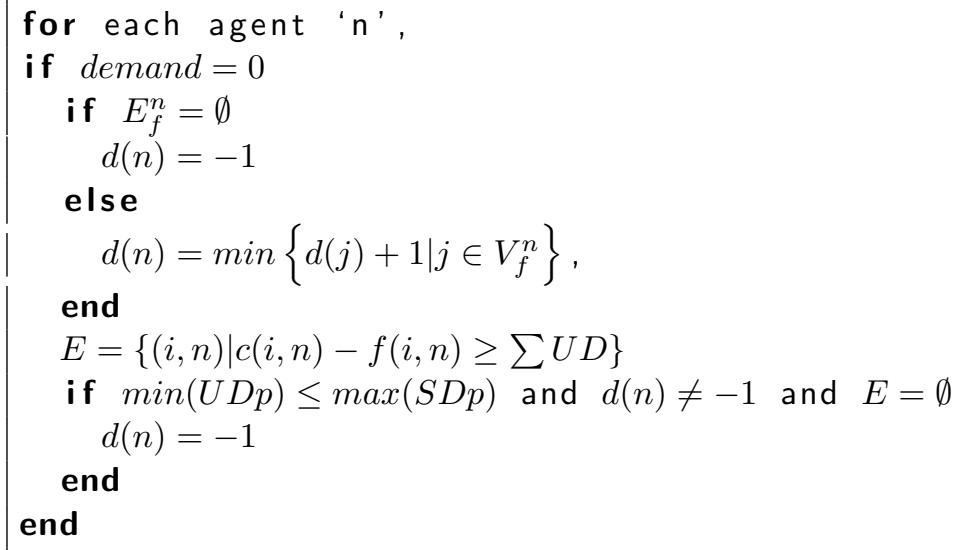

\section{GS2: Change Residue}

In maximum flow algorithm [28] residue is calculated as the difference between link capacity and link flow. The definition is modified to incorporate load priorities. The residue in this 
proposed algorithm is a function of unsupplied demand, residue of the incoming links, and load priority.

Each agent calculates residue if demand is zero. If the incoming flow over any bidirectional outgoing link is greater than zero, then the residue is set to zero. For all other outgoing links residue is sum of $\mathrm{R} 3$ and $\mathrm{R} 4$ :

$R_{2}$ is unsupplied demand on other links with higher unsupplied demand priority.

$R_{3}$ is maximum of residue on active incoming link and difference between maximum residue on non-active links and total outflow. It is the residual power available without removing any other load.

$R_{4}$ is the flow supplied to low priority loads over other active outgoing links. It is the residual power available based on priorities of loads.

Listing 3.2: GS2 Update residue

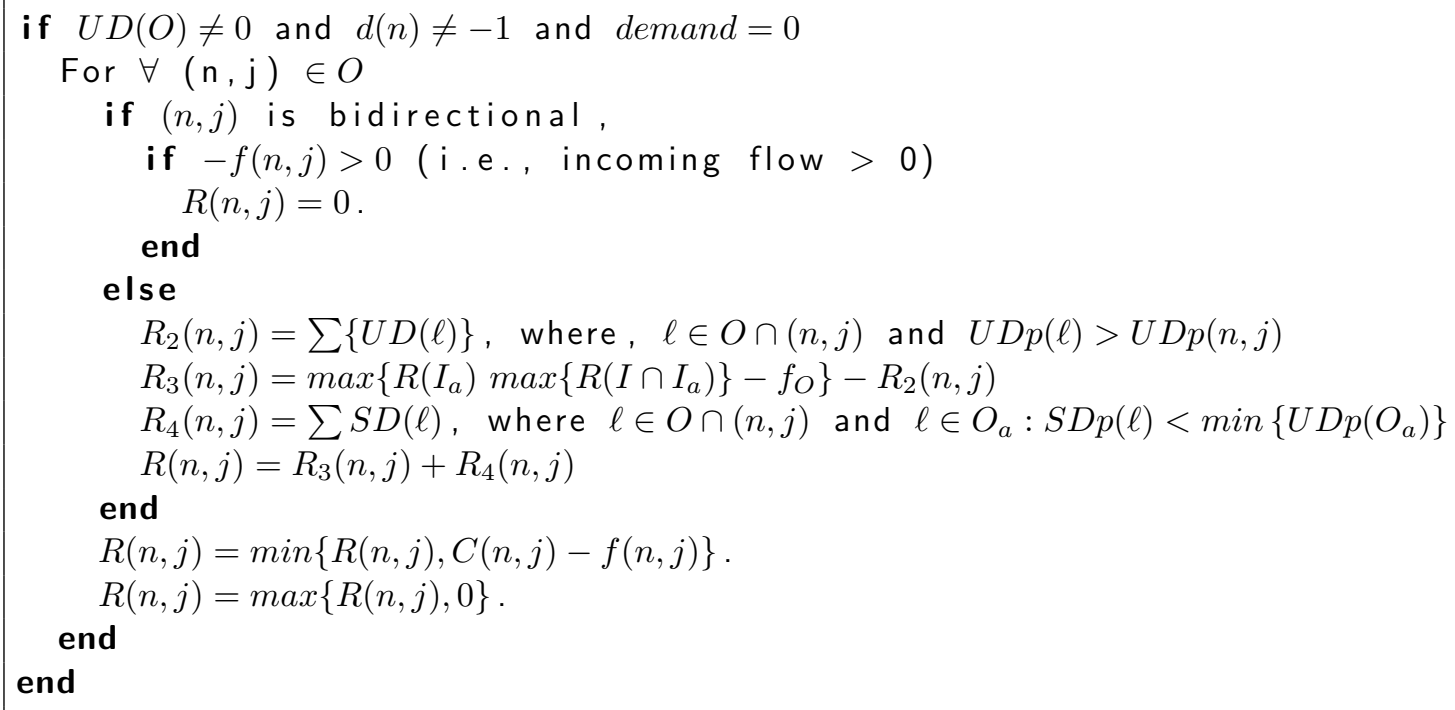

$R_{2}$ guaranties that high priority unsupplied demand is always satisfied. Agent assigns maximum available residual power to highest priority unsupplied demand. Consequently, residual power to other outgoing links is reduced by $R_{2}$. Thus $R_{3}$ represents maximum residue on a link $\ell$ from all input supply possibilities. $R_{4}$ represents any power available from shedding 


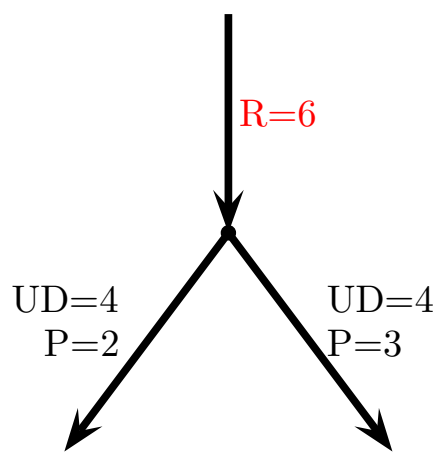

(a)

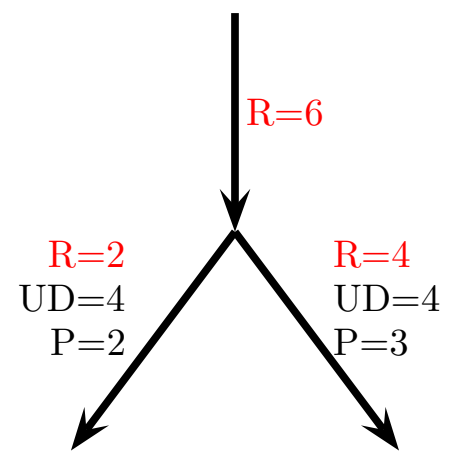

(b)

Figure 3.4: Example of residue assignment

low priority loads. It is the power supplied to other low priority loads compared to link $\ell$. Together, $R_{3}$ and $R_{4}$ represent maximum residual power available through reconfiguration.

Figure 3.4 gives an example of this statement. The agent has two outgoing links and one incoming link with residue 6 . The residue is divided into the two links according to the unsupplied demand priority. The high priority demand receives the required residual power, and other low priority load receives the remaining residue.

\section{GS3: Change Inflow}

Each agent executes this statement if demand is greater than zero. If active incoming link is present and residual power on that link is greater than demand then agent increases flow on that link. If residue over active link is less than demand, then agent finds an incoming link with residue greater than sum of demand and inflow, i.e., it checks for possibility to divert entire flow from one link to another. If such a link is present then it increases inflow on the new link by demand + inflow, and sets inflow of active incoming link to zero. Otherwise, it finds a link with maximum residue and increases flow over that link by residue. If there is no active incoming link, then agent increases flow over a link with maximum residue by minimum 
of residue and demand. An agent also changes residue values on the links to maintain data consistency.

Listing 3.3: GS3 Change Inflow

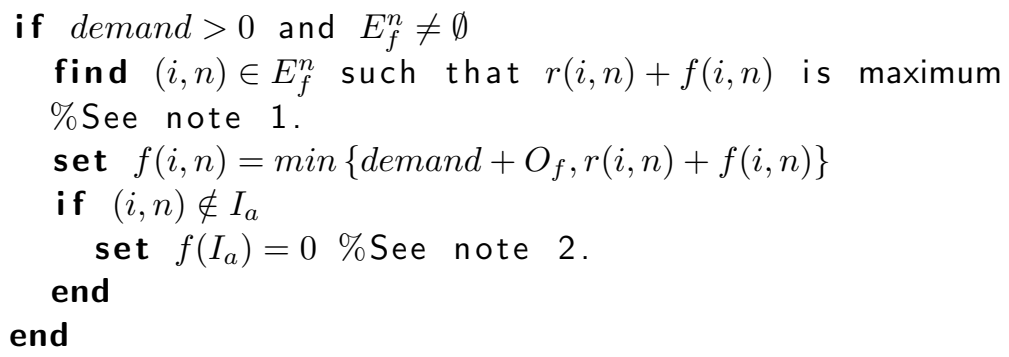

Note 1: When there are more than one link with maximum $r(i, n)+f(i, n)$, then any one link can be selected for further computation.

Note 2: This statement allows only one active incoming link. If it is possible to accept power from multiple sources simultaneously then this statement can be removed.

\section{GS4: Re-route Inflow}

With this rule an agent tries to find new incoming link so that the currently active incoming link gets some extra available power. Each agent with $\mathrm{d}=-1$ looks for optional incoming link with sufficient residual power and d-value greater than -1. If such a link exists, then the agent transfers entire incoming flow to the new link. If an agent reroutes the flow, it updates the priority information over those links, thus keeping the data consistent.

Listing 3.4: GS4 re-route inflow

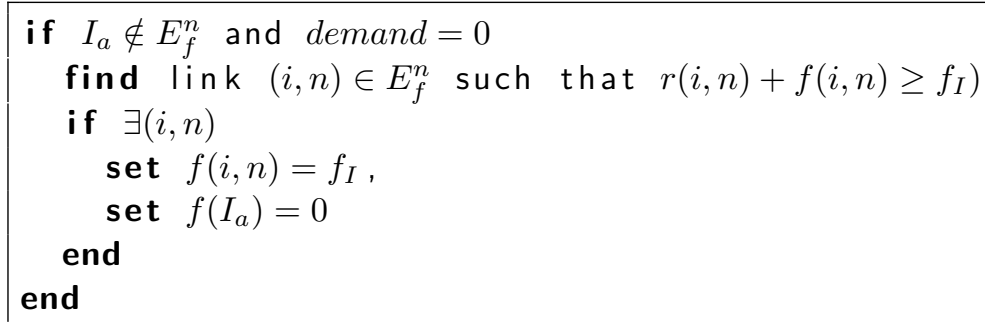




\section{GS5: Reduce Inflow}

If demand $<0$ then $\mathrm{f}\left(I_{a}\right)=\mathrm{f}\left(I_{a}\right)-\mid$ demand|. If inflow is greater than total outflow then the agent reduces inflow by amount equal to the difference.

Listing 3.5: GS5 Reduce Inflow

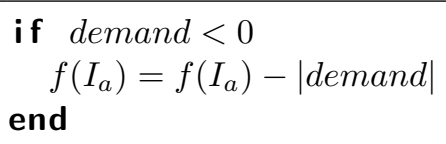

\section{GS6: Change Outflow}

If demand is greater than zero and no residue is available on incoming link, then find an outgoing link with lowest supplied demand priority and reduce outflow by minimum of flow and demand.

Listing 3.6: GS6 Change outflow

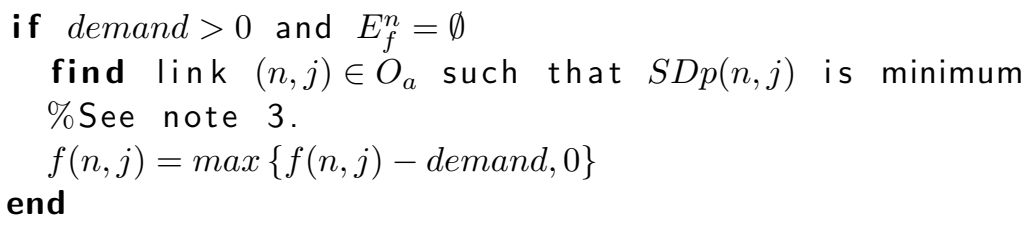

Note 3: If there are more than one outgoing links with minimum $S D p(n, j)$ then there are two options to proceed further. One option is to select any one link and reduce the flow over that link. This option is shown in above statements. Second option is to reduce the flow on all the links by equal amount.

\section{GS7: Change priority information}

When demand is zero, each agent updates the priority information over all incoming links. The information is updated according to their definitions:

$\operatorname{SDp}(\mathrm{i}, \mathrm{n})=$ Lowest priority value among all the active outgoing links. 
$\mathrm{SD}(\mathrm{i}, \mathrm{n})=$ Sum of supplied demand on all outgoing links with supplied demand priority $=$ $\operatorname{SDp}(\mathrm{i})$.

$\mathrm{UDp}(\mathrm{i}, \mathrm{n})=$ Highest priority of unsupplied demand.

$\mathrm{UD}(\mathrm{i}, \mathrm{n})=$ Sum of all unsupplied demands with priority UDp(i).

Listing 3.7: GS7 Change priority informantion

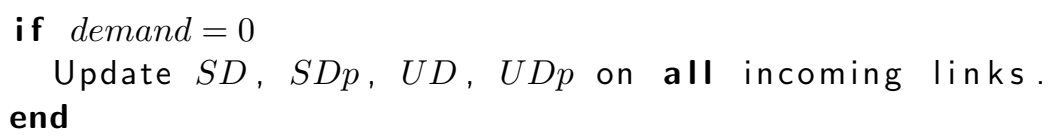

GS8: Remove capacity violation

If $\mathrm{f}(I)>\mathrm{C}(I)$ then $\mathrm{f}(I)=\mathrm{C}(I)$. Note that other statements never lead to this condition in case a valid initial flow existed. This statement rather allows changing system conditions including the loss of a link.

Listing 3.8: GS8 Remove capacity violation

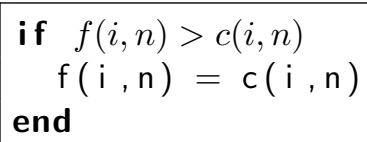

\subsection{Discussion}

Each agent independently executes these eight guarded statements and when an agent changes a variable, it informs other neighboring agents. When all agents finish the execution, the algorithm terminates. The residue value represents additional power available on that link. The residue is not the difference between maximum line capacity and line flow, but it considers load priorities, supplied, unsupplied demand and its priority on all outgoing links. Therefore, the residue can be less than the difference between line capacity and line flow. In this way, GS2 assigns residue on a link such that all high priority loads get required power. Every agent 
requests power by changing the unsupplied demand and its priority on the incoming links. Every agent tries to assign the residue values based on these unsupplied demands and their priorities. When an incoming link has non-positive residue, the agent increases the inflow to fulfill its power demand. In this way, the negotiation is initiated by load agent by changing UD and UDp, other agents respond to this request by assigning the required residue on outgoing links, and when the load agent sees required residue on the incoming link, it increases the inflow.

The original algorithm [27] considered a residual graph using the difference between link capacity and the flow. The algorithm does not consider any load priorities. In this new algorithm, the residue is computed based on load priorities. It is found that the new algorithm works better when it is required to consider the load priorities. All other guarded statements are modified according to the new concept of residual power.

When an agent $i$ cannot supply a low priority demand it changes its distance value to -1 . All agents on outgoing links of agent $i$ change their d-value to -1 . When an agent has d-value of -1 it tries to find alternative route to free some capacity of its present source. This action is performed by GS5. In other words, when an agent cannot supply a low priority load it requests other high priority loads to search for alternate paths and free some of its capacity to supply the low priority load.

\subsection{Conclusion}

This chapter explained a decentralized approach in solving the power system reconfiguration problem. The algorithm considered specific needs of shipboard power system that all high priority loads should be supplied first. The reconfiguration algorithm is executed by each agent and when all agents finish the execution, the agents send the new set points of line flows to implementation agents. 


\section{Chapter 4}

\section{Termination Detection}

All reconfiguration agents execute the guarded statements independently and share information via messages. When all agents finish the reconfiguration and find a solution, the agents become idle. If an agent knows that a consistent solution is present among all the agents and that all agents in the system agree with that solution it can inform the implementation agents about the changes required in device settings to activate the desired flow. Since every agent communicates only with its neighbors, it has a limited knowledge about other agents in the system. Therefore, an agent cannot determine that all agents in the system are idle and consistent solution has been found within the system. Therefore, it is necessary to design a procedure that will inform the reconfiguration agents about overall system status. This process of determining that all agents have finished the execution is known as termination detection. Termination detection in multiagent environment can be performed in two ways, centralized or distributed. 


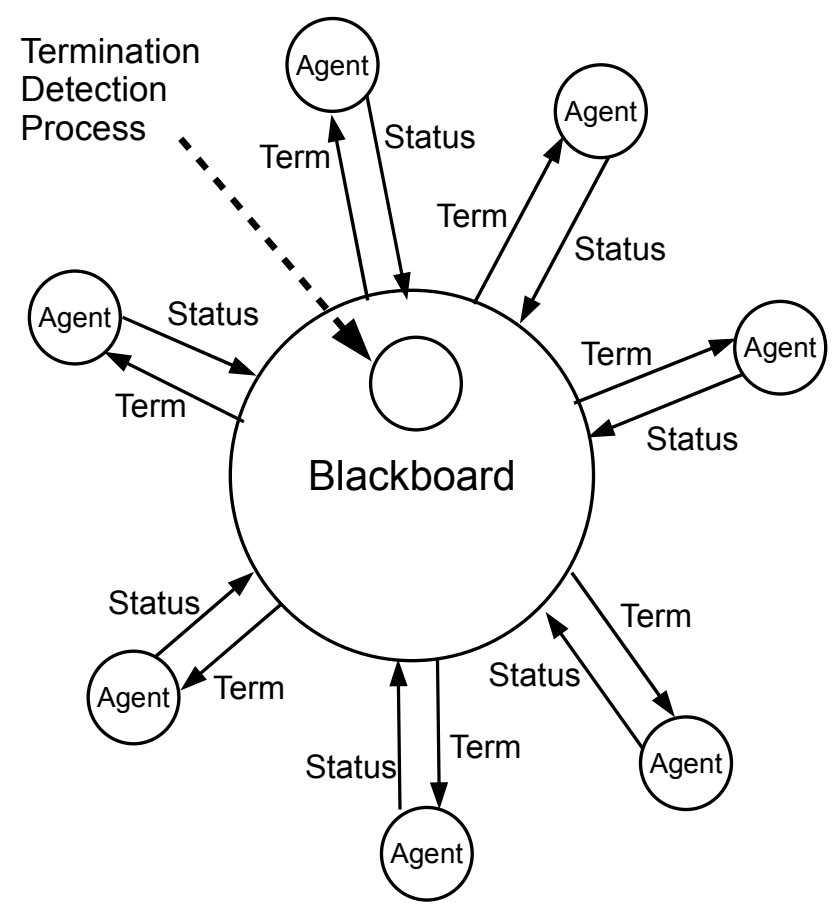

Figure 4.1: Blackboard concept

\subsection{Centralized Approach}

In the centralized termination detection, one process monitors the status of all agents in the system and decides whether all agents are idle [26]. Figure 4.1 shows a possible architecture for centralized approach. It consists of a shared memory, called blackboard, where all agents periodically post their status. The status is either 'idle' or 'executing'. One program known as 'Termination Detection Process' continuously checks the status of all agents. When it detects that all agents are idle the process sends a 'Term' message to all agents.

This approach contradicts with the very important property of agent based systems. Multiagent systems are designed for autonomous, independent and distributed decision making with small amount of communication. Therefore, the use of blackboard to determine the 


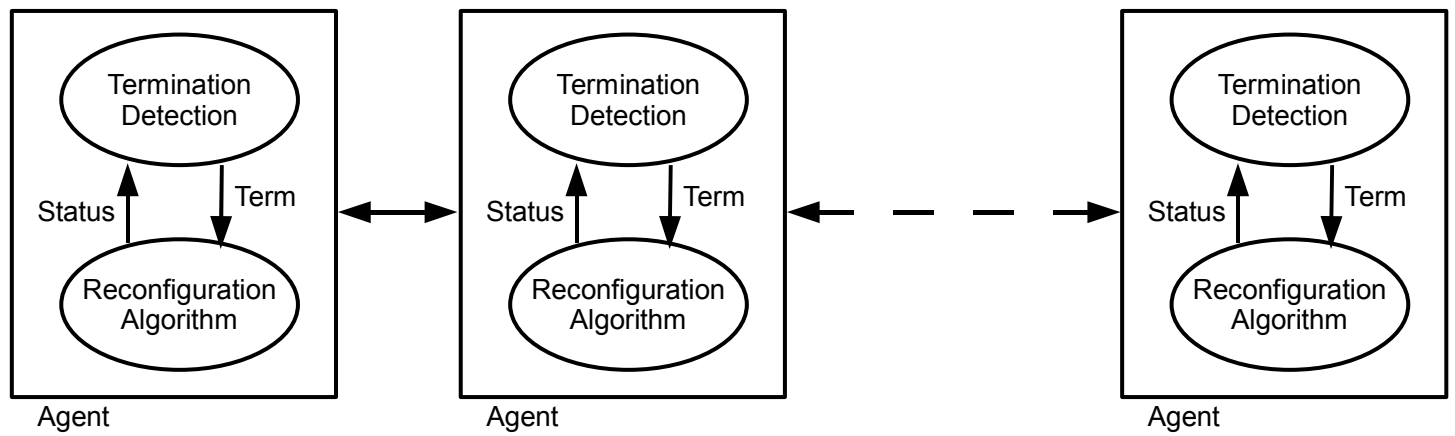

Figure 4.2: Concept of distributed termination detection

algorithm termination violates this distributed decision making concept. Though, the communication between agents and blackboard is small, all agents are dependent on one single process for the final decision. The agent based approach has been selected to improve survivability and damage to one agent should not affect entire reconfiguration system. In the centralized approach the blackboard is a single point of failure, i.e., failure of the blackboard will put entire reconfiguration algorithm into jeopardy, and none of the agents will be able to terminate. Therefore, the use of blackboard for such an important task is not justifiable and some other method is required.

\subsection{Distributed Approach}

The distributed approach is designed to avoid the single point of failure. In this method, all agents contribute to the decision and every agent learns about the global termination through a local termination detection process. The concept is explained in Figure 4.2. Some examples of termination detection algorithms used in distributed computing are explained in Chapter 2 .

\section{Important concepts}

Basic algorithm: Reconfiguration Algorithm. It is the algorithm whose termination is 
to be detected.

Basic messages: Messages exchanged by reconfiguration algorithm.

Control algorithm: The termination detection algorithm.

Control messages: Messages exchanged for termination detection.

\subsection{Proposed Algorithm}

The termination detection algorithm for the shipboard power system reconfiguration is based on the wave algorithm as explained in Chapter 2. In the original wave algorithm, one agent initiates the wave computations and when wave returns. the initiator makes the decision. Therefore, only one agent is able to detect the termination. To satisfy the requirement of the SPS reconfiguration algorithm, the original wave algorithm is extended by using features found in [24].

The control algorithm uses the same graph structure from the basic algorithm and forms multiple logical spanning trees (4.3.1). The wave algorithm is initiated by each root and spreads in downward direction to the leaves and then back upwards to the root. When an intermediate node receives the wave from all of its children then it sends the accumulated wave to its parent. Each node follows certain rules (4.3.2) while sending the wave signal. In summary, the rules allow every agent to detect the termination when it sends two consecutive white waves. In this algorithm the wave computations do not terminate but continue forever to allow continuous detection and response to the changing environment of the reconfiguration algorithm. 


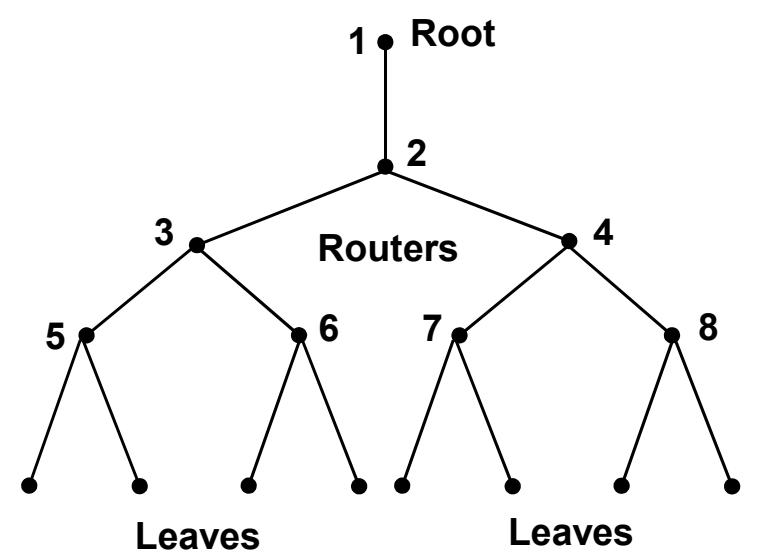

(a) Example Tree

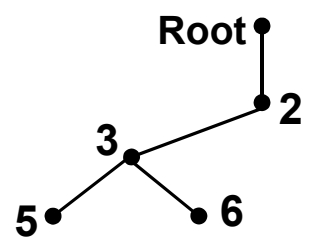

Node 3: Parent $\{2\}$ Children $\{5,6\}$

Figure 4.3: Example tree and parent and children list

\subsubsection{Spanning Forest}

The underlying construction of the spanning forest is based on breadth first search [28]. This algorithm forms multiple rooted trees at the start of the system and preserves them unless changes in physical connections occurs viz. loss of link, loss of an agent, new link, new agent, etc. A distance value (d-value) of a node is defined similar to reconfiguration algorithm as the number of links from the root to the node. Though the definition is same as reconfiguration d-value, the two variables are different and independent.

The d-value of root node is always zero. Each agent holds a unique parent node and a list of children nodes. Initially, the list of children and parent is empty and the d-values of all nodes except roots are set to a high number, e.g., total number of nodes in the system or diameter of the graph. Every node $n$ compares the d-values of its neighbors and selects a node $k$ with the lowest $\mathrm{d}$-value $d_{k}$ as its parent. It changes its local d-value to $d_{k}+1$ and informs node $k$ to update the list of children. Root node starts the wave algorithm as soon as it receives first child by sending a white wave to it. When a wave visits an agent for the first time, the agent freezes its parent and becomes part of the tree. If the agent has children 
then it forwards the wave to them, otherwise it sends it back to the parent. Thus eventually all nodes receive a wave, fix their parent and hold a list of children. When an agent freezes its position in a tree, it starts the basic computations. When the wave returns to the root, it starts a new wave using rules specified in the following section.

\subsubsection{Rules}

Every agent processes the wave using certain rules and follows a state transition diagram. The wave computation flows through the system using a color variable ' $C$ ' (equation 4.1). Black color means that at least one agent is executing basic algorithm in the wave's path and White color means that all agents in the wave's path are idle.

$$
C \in\{\operatorname{Black}(B), \text { White }(W)\}
$$

Next, we define agent states $Z$ as

$$
Z \in\{\operatorname{Active}(A), \text { Idle }(I), \text { Terminated }(T)\}
$$

Any agent becomes Active when it executes a guarded statement, receives a basic message or receives a black wave. An agent is Idle when it has no guarded statement to execute and has forwarded a white wave. Any agent that forwards white wave consecutively two times detects global termination and becomes Terminated.

\section{Rules:}

1. Every agent that receives a wave sends a Black wave if the received wave is Black or it currently performs any basic computation or received a basic message after sending the last wave.

2. Every agent that receives a wave sends a White wave if the received wave is White and 
it did not perform a basic computation or did not receive a basic message after sending the last wave.

3. Any agent that sends a Black wave becomes Active.

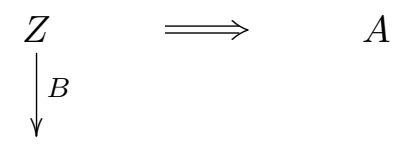

4. Any Active agent that sends a White wave becomes Idle. The Idle state signifies a partial termination. The node believes that all other agents have finished basic computation and it waits for the confirmation, i.e., another White wave.

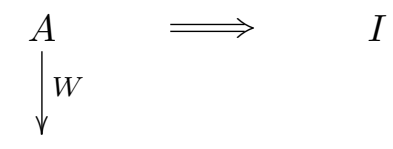

5. Any Idle agent that sends a White wave terminates.

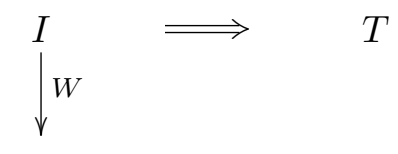

6. Any intermediate agent that receives a wave from the parent sends out a wave to all its children by following the above rules.

7. Any intermediate agent that receives waves from all its children sends a wave to the parent by following the above rules.

8. Any leaf agent that receives a wave from its parent sends a wave to its parent following the above rules. 


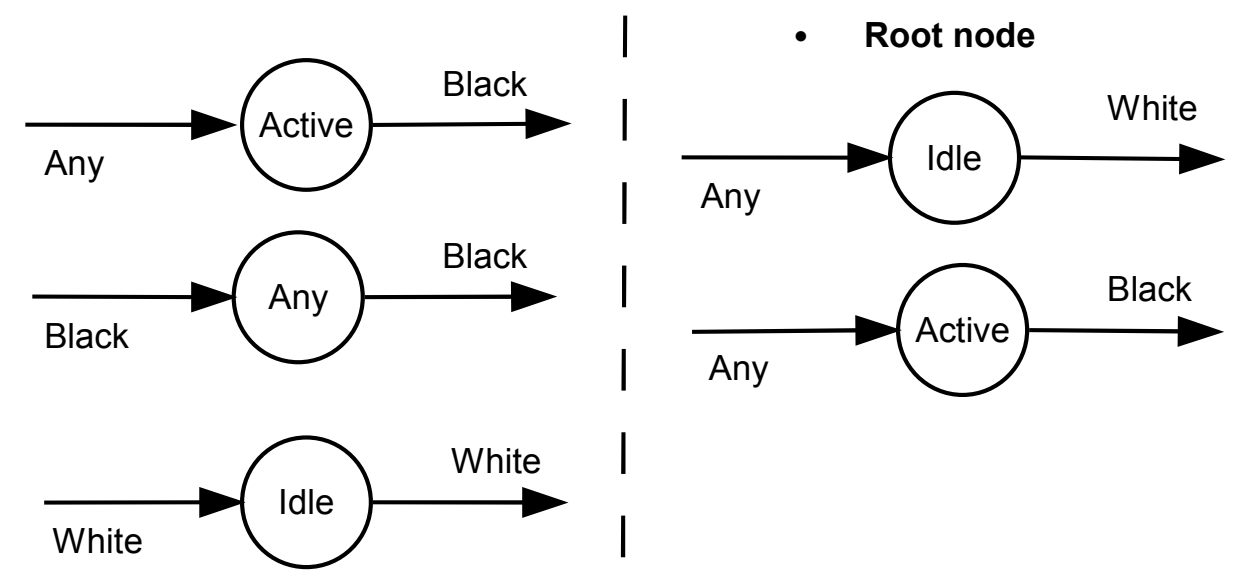

Figure 4.4: Graphical representation of rules one, two and nine.

9. If a root receives a wave it follows the above rules to make a decision and change its status. If the root agent is active then it sends out a black wave, otherwise it sends a white wave.

Rules one, two and nine are represented in Figure 4.4, Figure 4.5 shows the state diagram for the control algorithm using rules three, four and five. Every agent follows these rules and processes the wave. Since the basic computation, i.e., the maximum flow algorithm is itself stable and eventually reaches termination; it is guaranteed that the wave algorithm will detect the termination.

\subsection{Discussion}

In the reconfiguration algorithm it is necessary to communicate the final termination decision to all agents in the system. This situation is comparable to one central process detecting termination and informing the other agents. This problem is addressed by this wave algorithm through the propagation of two consecutive white waves and allows each node to locally decide on termination. The existence of multiple trees according to the number of available sources 


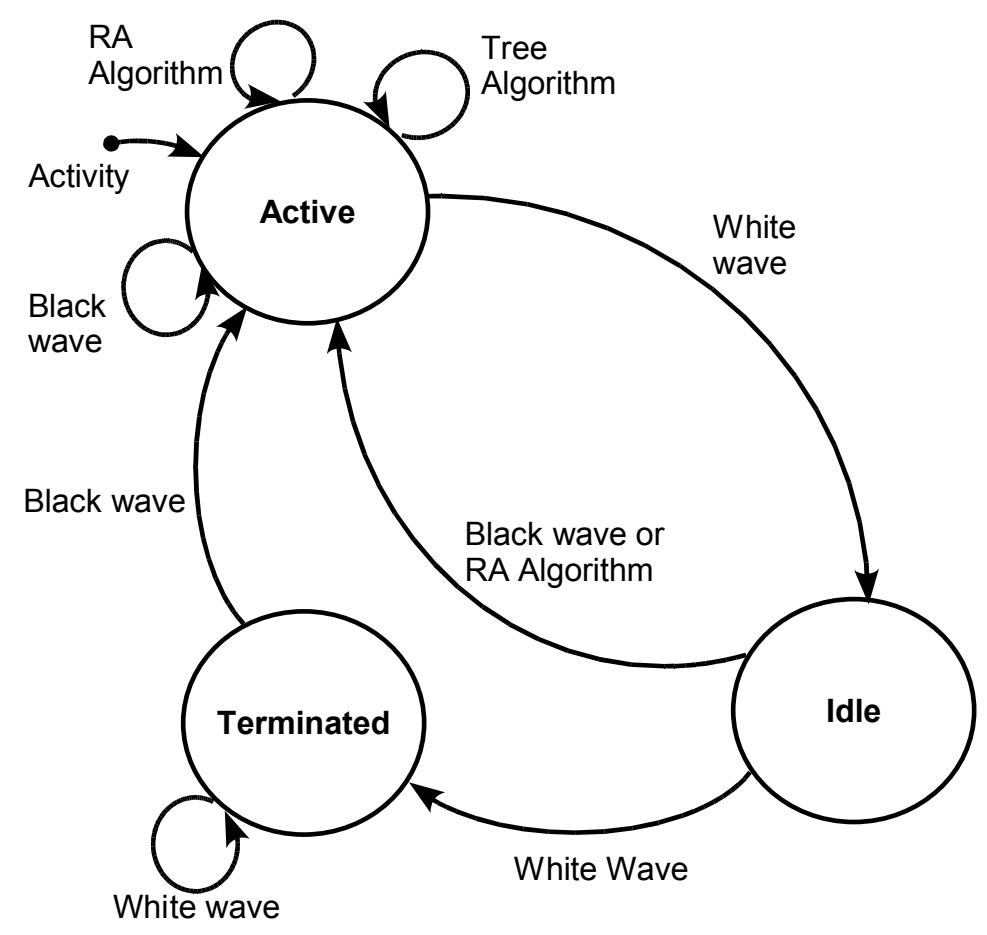

Figure 4.5: State diagram, where the Black and White waves denote sending events.

ensures that load demands are supplied as long as these are reachable from the remaining sources.

In implementing the algorithm, it is also important to ensure that all basic computations and messages are given higher priority than the wave algorithm and associated messages. Otherwise, the wave algorithm will simply prevent execution of basic computation by an agent and it will flood the communication channels and consequently prevent the basic algorithm from proceeding.

Termination detection algorithm is followed by actual implementation of the solution. When an agent detects termination it translates the reconfiguration solution into control signals and sends the new settings to the respective implementation agent. Implementation agents explained in 6.2 control the hardware (converters, breakers, and generator) to realize the new reconfiguration solution. 


\section{Chapter 5}

\section{Communication}

Communication is an inherent property of multiagent systems. All agents need to communicate with their neighbors to achieve their local goals as well as global objectives. The agents require that the communication interface is error free and reliable. Agents also require a common language, a message structure and uniform addressing scheme to talk to each other. This chapter provides such a scheme for agent communication over CAN bus. The purpose of this scheme is to provide a message structure and database using FIPA Agent Communication Language (ACL) and CANOpen data structure.

Agent system consists of a "Command and Control Center" (CCC), communication backbone and individual devices [27]. A device is a controller unit that can represent a reconfiguration agent, an implementation agent, a measurement device, etc. The command and control center (Section 6.4) is responsible for monitoring information flow, providing user interface and providing supervisory control over the system.

The literature survey gave an overview of FIPA agent architecture and agent communication structure. These recommendations are used in this structure with mandatory specifications by FIPA while designing the communication architecture. Specifically, the object dictionary is compliant with CANOpen and the message structure and agent structure given 


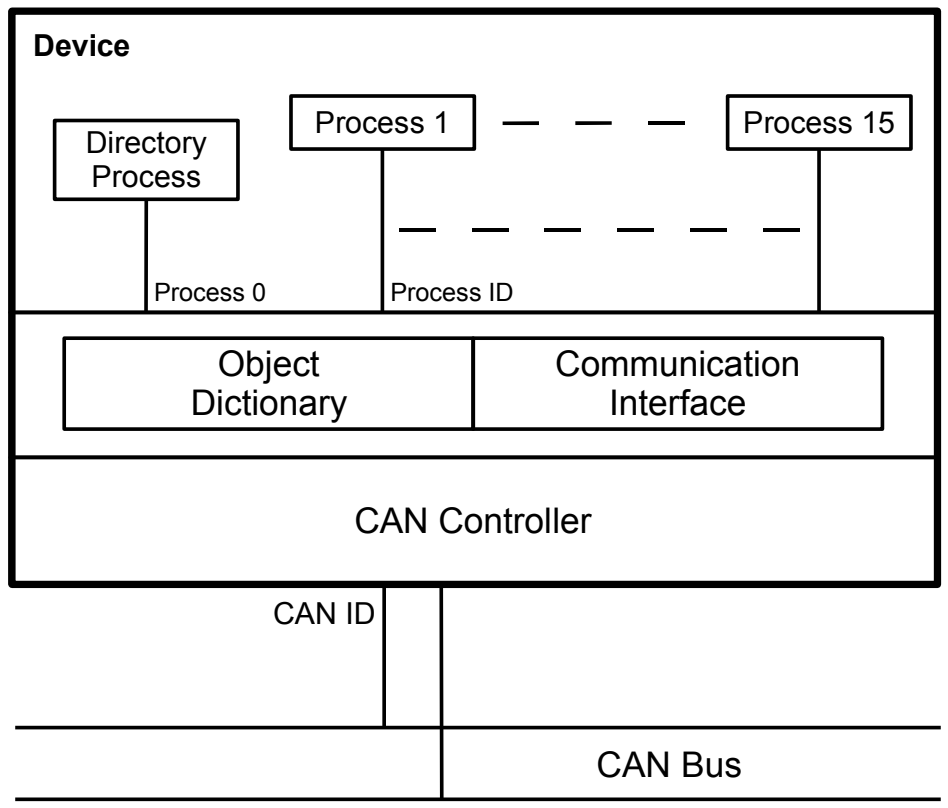

Figure 5.1: Device architecture

in Figure 5.1 is recommended by FIPA [56].

\subsection{System Architecture}

Device [62]: It is any micro-controller, microprocessor, computer connected to CAN bus. In SPS, these devices can represent implementation agents, reconfiguration agents, measurement devices or command and control center. These devices can have multiple CAN addresses but only one physical interface. Each CAN address will represent one logical connection. At startup each device will have one unique CAN address programmed into it. Provision can be made to change this address when device connects to the network [48]. One CAN address can be used by fifteen processes. When there are more processes running on a device, another CAN address can be acquired from command and control center or can be preprogrammed in the device. 
Process: It is a program running on the device. A device has multiple programs running on it. For example, multiple reconfiguration agents or reconfiguration agent and implementation agent, etc. The addressing scheme allows sixteen processes for one logical connection. Thus, one CAN ID can support 16 processes (agents). If a device has more than 16 processes running, it can establish more than one logical connection with different CAN IDs. A Process ID that is local to that device [56] identifies each process.

Agent name: This is the name of the process represented with 4 bytes. These names are unique in the complete shipboard power system, e.g., motor controller 1 (MC1), Constant power load 1 (CPL1), etc. CAN ID followed by a process ID identifies an agent. Command and control center holds the complete reference table for agent name, CAN ID and Process ID. Each device also has a directory agent that facilitates the communication.

Directory process (DP)[56]: Agent communication network requires directory service for agent names, their CAN IDs, process IDs. These entries can be updated by Command and Control Center. In the implementation of the multiagent system a separate directory process is recommended by FIPA to provide the directory service to other agents on a device. FIPA also recommends a fixed Process ID of zero (0h) for directory process.

Communication interface: It is an entity on each device that translates the message requests and process data into the CAN bus message format. Whenever a process wants to communicate, it invokes the communication interface. This interface generates the messages, adds required fields like process IDs, and creates CAN identifier and frame numbers. Afterwards, the message is sent on the CAN bus through CAN controller [59].

CAN controller: CAN controller handles the communication over CAN bus. It performs all the tasks mentioned in the CAN protocol [8]. These are error handling, message filtering, CAN bus timings, bit stuffing, etc. 
Frame and Message: A frame is a message with up to eight bytes of data. The communication protocol allows for a maximum of 255 frames per message.

Floating point standard : The IEEE single precision floating point standard representation requires a 32-bit word, numbered from 0 to 31, left to right. The first bit is the sign bit, $\mathrm{S}$, the next eight bits are the exponent bits, ' $\mathrm{E}$ ', and the final 23 bits are the fraction ' $\mathrm{F}$ ': [5]

\section{S EEEEEEEE FFFFFFFFFFFFFFFFFFFFFFF}

$\begin{array}{lll}01 & 89\end{array}$

The value $\mathrm{V}$ represented by the word may be determined as follows:

If $\mathrm{E}=255$ and $\mathrm{F}$ is nonzero, then $\mathrm{V}=\mathrm{NaN}$ ("Not a number")

If $\mathrm{E}=255$ and $\mathrm{F}$ is zero and $\mathrm{S}$ is 1 , then $\mathrm{V}=$-Infinity

If $\mathrm{E}=255$ and $\mathrm{F}$ is zero and $\mathrm{S}$ is 0 , then $\mathrm{V}=$ Infinity

If $0<\mathrm{E}<255$ then $V=(-1)^{S} * 2^{(E-127)} *(1 . F)$ These are normalized values. Where "1.F" is intended to represent the binary number created by prefixing $\mathrm{F}$ with an implicit leading 1 and a binary point. For normalized floating point representation the range of number is approximately $\pm 10^{38}$ to $\pm 10^{-38}$.

If $\mathrm{E}=0$ and $\mathrm{F}$ is nonzero, then $V=(-1)^{S} * 2^{-126} *(0 . F)$ These are de-normalized values.

If $\mathrm{E}=0$ and $\mathrm{F}$ is zero and $\mathrm{S}$ is 1 , then $\mathrm{V}=-0$.

If $\mathrm{E}=0$ and $\mathrm{F}$ is zero and $\mathrm{S}$ is 0 , then $\mathrm{V}=0$.

\section{$5.2 \quad$ Message Structure}

This section gives details of all messages required for agent communication for SPS reconfiguration agents. This structure has been designed using bit-efficient protocols given by FIPA 
$[61,60]$. The CAN bus provides data field of only 8 bytes, therefore this message structure considers only some fields specified in FIPA messages but considers all the mandatory parameters given in $[58,55]$.
Digit*
Represents zero or multiple repetitions of digit.
$0 x ? ?$
Represents byte in hexadecimal

\begin{tabular}{|c|c|c|}
\hline ACLCommunicativeAct & $=$ & Message \\
\hline Message & $=$ & $\begin{array}{l}\text { CAN_Identifier Data_Field } /{ }^{*} \text { CAN Identifier is } 29 \text { bit and } \\
\text { CAN data field is } 8 \text { byte* } /\end{array}$ \\
\hline CAN_Identifier & $=$ & Priority | From | To | FrameBit \\
\hline Data_Field & $=$ & FrameID FromProcess ToProcess MsgAct MsgContent \\
\hline Priority & $=$ & Nibble $/ * 16$ different Priorities, Table 5.3*/ \\
\hline From & $=$ & Device Address \\
\hline To & $=$ & Device Address \\
\hline Device Address & $=$ & Nibble Nibble Nibble. $/{ }^{*} 2^{12}=4096$ devices. */ \\
\hline FrameBit & $=$ & $\begin{array}{l}0 / * \text { This is last frame of the message } * / \\
1 / * \text { This is intermediate frame and FrameID is the frame } \\
\text { number. } * /\end{array}$ \\
\hline FrameID & $=$ & Byte /* Total 256 frames are possible per message. */ \\
\hline FromProcess & $=$ & ProcessID \\
\hline ToProcess & $=$ & ProcessID \\
\hline ProcessID & $=$ & $\begin{array}{l}\text { Nibble } / * \text { Since there are } 2^{12}=4096 \text { Device IDs and } 16 \\
\text { Process IDs total processes/agents available in the system } \\
\text { are } 4096 * 16=65,536 . *\end{array}$ \\
\hline MsgAct & $=$ & $\begin{array}{l}\text { PredefinedMsg } / *[57] * / \\
\text { UserdefinedMsg }\end{array}$ \\
\hline
\end{tabular}




\begin{tabular}{|c|c|c|}
\hline MsgContent & $=$ & Proposition BinString \\
\hline & | & BinString \\
\hline BinString & $=$ & $(\text { Byte })^{*}$ \\
\hline & $\mid$ & NewValue \\
\hline & $\mid$ & Reason \\
\hline PredefinedMsg & $=$ & 0x01 /* Accept-proposal */ \\
\hline & $\mid$ & $0 \mathrm{x} 03 / *$ Cancel $* /$ \\
\hline & | & 0x05 /* Confirm */ \\
\hline & $\mid$ & 0x06 /* Disconfirm */ \\
\hline & | & 0x08 /* Inform */ \\
\hline & $\mid$ & 0x0d /* Propose $* /$ \\
\hline & $\mid$ & 0x0f /* Queryif */ \\
\hline & | & 0x13 /* Request */ \\
\hline UserdefinedMsg & $=$ & 0x20/* Quit */ \\
\hline & $\mid$ & $0 \times 21 / *$ Halt $* /$ \\
\hline & $\mid$ & $0 \times 22 / *$ Add $* /$ \\
\hline & $\mid$ & $0 \times 23 / *$ Reset $* /$ \\
\hline & $\mid$ & 0x24/* Solution Converged */ \\
\hline Propositions & $=$ & $0 \mathrm{x} 1 \mathrm{~m} / *$ Cval (Line capacity) $\mathrm{m}=1$ for maximum capacity \\
\hline & & and $\mathrm{m}=2$ for minimum capacity $* /$ \\
\hline & $\mid$ & 0x02 /* Dval (Distance value) $* /$ \\
\hline & $\mid$ & 0x03 /* Fval (Line flow) */ \\
\hline & $\mid$ & 0x04 /* Pause */ \\
\hline & $\mid$ & 0x05 /* Resume */ \\
\hline & $\mid$ & $0 \mathrm{x} 06 / *$ Power $* /$ \\
\hline & $\mid$ & 0x07 /* Error */ \\
\hline & 1 & 0x08 /* NewIndex */ \\
\hline
\end{tabular}


| $0 \mathrm{x} 9 \mathrm{~d} / *$ DirectoryEntry, $\mathrm{d}=0$ indicates the inquiry, $\mathrm{d}=1$ indicates the response for $1^{\text {st }}$ index requested. $\mathrm{d}=2$ indicates the response for $2^{\text {nd }}$ index requested. */

| $0 \mathrm{x} 0 \mathrm{a} / *$ Device Address */

| $0 \mathrm{x} 0 \mathrm{~b} / *$ AgentName*/

| $0 \mathrm{x} 0 \mathrm{c} / *$ Parent */

| $0 x 0 d / *$ Pval (Priority) */

| $0 \mathrm{x} 0 \mathrm{e} / *$ Residue */

| 0xex /* Pmtx (Priority Matrix/ Priority Quadruple)*/

| $0 \mathrm{x} 0 \mathrm{f} / * \operatorname{Birth} * /$

| $0 \times 10 / *$ Status */

| $0 x 11 / *$ Wave color */

| $0 \times 12 / * \mathrm{~d}$-value of termination detection algorithm*/

| $0 \times 1 b / *$ Controller set point for simulink */

Reason $\quad=$ PreDefinedErrorMsg $/ *$ Appendix A*/

| NULL

NewValue

$=$ SinglePrecesion $/ *$ IEEE32 floating point number */

| UINT32 /*32 bit unsigned integer */

\subsection{Message Definitions}

1. Inform message : This message is sent by an agent or command and control center when it wants to report a change in value of a variable. An agent also sends this message as a response to 'enquiry' message. 'Proposion' field specifies the variable name. The NewValue is absolute value of the variable, i.e., it is not specified as a change in value. Example: Informing the d-value of reconfiguration algorithm.

Inform (08) d-value (02) NewValue 


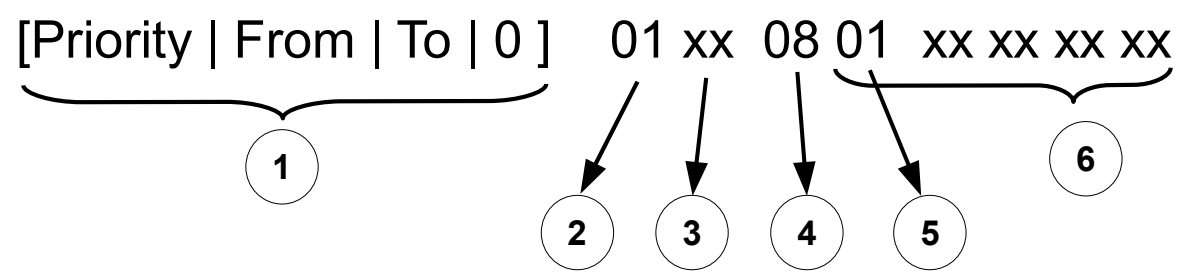

1. CAN Identifier

3. [FromProcess] [ToProcess]

5. Proposition
2. FrameID

4. MsgAct

6. MsgContent

Figure 5.2: Message structure example

[Priority|From|To|0] $01 \mathrm{xx} 0802 \mathrm{xx} \mathrm{xx} \mathrm{xx} \mathrm{xx}$

2. Enquiry message : This message is sent by an agent or command and control center when it wants to know the value of another agent's variable. The variable name is specified in 'proposion' field.

Example : Inquiring the d-value of reconfiguration algorithm.

Request(13) d-value(02)

[Priority|From|To|0] 01 xx 1302

3. Delete Link : This message can be sent by implementation agent or command and control center to reconfiguration agent. This message uses 'Cancel' message action and a set of agents specifies the link.

Cancel(03) Agent1DeviceID (12 bit) Agent1ProcessID (4 bit) Agent2DeviceID (12 bit) Agent2ProcessID (4 bit)

[Priority|From|To|0] 01 xx 03 xxx x xxx x

4. New Link : This message can be sent by command and control center to reconfiguration agent. This message uses 'Add' message action and the link is specified similar to link delete message. 
Add(22) Agent1DeviceID (12 bit) Agent1ProcessID (4 bit) Agent2DeviceID (12 bit) Agent2ProcessID (4 bit)

[Priority|From|To|1] 01 xx 22 xxx x xxx x

5. Power Demand : Power requested by implementation agent or load to the reconfiguration agent. This message triggers the maximum flow algorithm.

Queryif(0f) Power(06) NewValue.

[Priority $\mid$ From $\mid$ To $\mid 0] 01$ xx 0f 06 xx xx xx xx

6. New Power : Reconfiguration agent sends this message after completing the maximum flow algorithm. The message informs the implementation agent about the new power setting.

$\operatorname{Inform}(08)$ Power(06) NewValue

[Priority|From|To|0] 01 xx 0806 xx xx xx xx

7. Power Reply : The reconfiguration agent sends this message to load after completing the maximum flow algorithm. The reconfiguration agent can accept complete requested flow or some part of it. The new value will be sent as NewValue.

Confirm(05) Power(06) OR

Disconfirm(06) Power(06) NewValue.

[Priority|From|To|0] 01 xx 0506 OR

[Priority|From|To|0] 01 xx 0606 xx xx xx xx

\section{Error Message :}

Inform(08) Error(07) PreDefinedErrorMsg.

[Priority|From|To|0] 01 xx 0807 xx xx

9. Pause message: When an error occurs in the execution, an agent sends this message to command and control center. The agent informs that it paused because of error 
specified in PreDefinedErrorMsg. In formal ACL language, this can be explained as sender proposes to pause provided that the receiver will understand the error and try to remove the error.

Propose(0d) Pause(04) PreDefinedErrorMsg.

[Priority|From|To|0] 01 xx 0d 04 xx xx xx xx

10. Resume message: This message is sent by an agent to command and control center to ask for permission to resume normal operation.

Propose(0d) Resume(05).

[Priority $\mid$ From $\mid$ To $\mid 0] 01$ xx 0d 05

11. Allow Resume: This message is sent by command and control center in response to 'Resume Message'. When CCC receives resume message, either user can allow the device to resume or CCC itself analyzes any dependencies and allows the device to resume its normal operation.

Accept-proposal(01) Resume(05)

[Priority $\mid$ From $\mid$ To $\mid 0] 01$ xx 0105

12. Quit: This message is sent by an agent when it cannot perform any operation due to some error and has to shut down itself. Once quit, the device may not receive any messages over CAN bus.

Quit(20) Error(07) PreDefinedErrorMsg.

[Priority|From|To|0] 01 xx 2007 xx xx xx xx

13. Halt: Command and control center sends this message to stops execution of all the agents on the network.

$\operatorname{Halt}(21)$

[Priority|From|To|0] 01 xx 21 
14. Reset: Command and control center sends this message to reset all devices on the network. This message works only with controllers with software reset capability.

$\operatorname{Reset}(23)$

[Priority|From|To|0] 01 xx 23

15. Resume: To resume the system from halt state. This message is sent by command and control center.

Inform(08) Resume(05)

[Priority|From|To|0] 01 xx 0805

16. Birth message: The message will contain the agent name.

Inform(08) Birth(0f) AgentName

[Priority|From|To|0] 01 xx 08 0f xx xx xx xx

17. Status message: This message informs activity status of the device. It is sent periodically with fixed time interval. State is the operating condition of the device according to the state diagram.

$\operatorname{Status}(08) \operatorname{Status}(10)$ state

[Priority|From|CCC|0] 01 xx 0810 xx

\subsection{Addressing Scheme}

In the CAN protocol, CAN identifier decides the message arbitration. If two or more devices start transmitting messages at the same time, the bus access conflict is resolved by bit-wise arbitration using the identifier [8]. During arbitration, every transmitter compares the level of the bit transmitted with the level of the received bit. If these levels are equal, the device may continue to send the message. When a "recessive" level is sent and a "dominant" level is monitored, the unit has lost arbitration and must withdraw without sending one more bit. In 
this way, the priority is given to the "dominant" level, which is a zero. Thus in the arbitration the message having lower CAN identifier wins the arbitration.

In the shipboard power system the command and control center is most important and thus it is given highest priority, i.e., zero address. The next address range allotted as special devices, might be required during ship maintenance and repair operations to connect handhelds and portable units. All the other devices are allotted the remaining address space. The devices are classified according to their distance from main generators in the SPS. The address range covers all the devices related to this class, e.g., AC generator class covers the generator controllers as well as the reconfiguration agents for generator, any measurement devices for the generator, etc.

Any change made at the generator level affects the complete system. If a generator and some other device are sending a message of same priority, then it is important that the generator should get priority over other device. Thus, generators use lower address levels and higher addresses are assigned to loads. Similarly, all other devices use intermediate addresses according to their classes. Table 5.2 gives the addressing scheme suggested for shipboard power system.

Table 5.2: Addressing scheme

\begin{tabular}{|c|c|c|c|c|c|c|c|c|c|c|c|c|c|c|c|}
\hline \multirow{2}{*}{$\begin{array}{l}\text { Class } \\
\text { CCC } \\
\end{array}$} & \multicolumn{12}{|c|}{ Address range } & \multicolumn{2}{|c|}{$\begin{array}{l}\text { Address } \\
\text { range in } \\
\text { Hexadecimal }\end{array}$} & \multirow{2}{*}{$\begin{array}{c}\begin{array}{l}\text { No. of } \\
\text { devices }\end{array} \\
1 \\
\end{array}$} \\
\hline & 0 & 0 & 0 & 0 & 0 & 0 & 0 & 0 & 0 & 0 & 0 & 0 & $000 \mathrm{~h}$ & & \\
\hline Blackboard & 0 & 0 & 0 & 0 & 0 & 0 & 0 & 0 & 0 & 0 & 0 & 1 & $001 \mathrm{~h}$ & & 1 \\
\hline Special devices & 0 & 0 & 0 & 0 & 0 & 0 & 1 & $\mathrm{X}$ & $\mathrm{X}$ & $\mathrm{X}$ & $\mathrm{X}$ & $\mathrm{X}$ & $020 \mathrm{~h}$ & $03 \mathrm{Fh}$ & 32 \\
\hline AC Generator & 0 & 0 & 0 & 0 & 0 & 1 & $\mathrm{X}$ & $\mathrm{X}$ & $\mathrm{X}$ & $\mathrm{X}$ & $\mathrm{X}$ & $\mathrm{X}$ & $040 \mathrm{~h}$ & $07 \mathrm{Fh}$ & 64 \\
\hline Power Supply & 0 & 0 & 0 & 0 & 1 & $\mathrm{X}$ & $\mathrm{X}$ & $\mathrm{X}$ & $\mathrm{X}$ & $\mathrm{X}$ & $\mathrm{X}$ & $\mathrm{X}$ & $080 \mathrm{~h}$ & 0FFh & 128 \\
\hline DC Bus & 0 & 0 & 0 & 1 & 0 & $\mathrm{X}$ & $\mathrm{X}$ & $\mathrm{X}$ & $\mathrm{X}$ & $\mathrm{X}$ & $\mathrm{X}$ & $\mathrm{X}$ & $100 \mathrm{~h}$ & $17 \mathrm{Fh}$ & 128 \\
\hline Converter & 0 & 0 & 0 & 1 & 1 & $\mathrm{X}$ & $\mathrm{X}$ & $\mathrm{X}$ & $\mathrm{X}$ & $\mathrm{X}$ & $\mathrm{X}$ & $\mathrm{X}$ & $180 \mathrm{~h}$ & $1 \mathrm{FFh}$ & 128 \\
\hline Converter & 0 & 0 & 1 & $\mathrm{X}$ & $\mathrm{X}$ & $\mathrm{X}$ & $\mathrm{X}$ & $\mathrm{X}$ & $\mathrm{X}$ & $\mathrm{X}$ & $\mathrm{X}$ & $\mathrm{X}$ & $200 \mathrm{~h}$ & $3 \mathrm{FFh}$ & 512 \\
\hline Load & 0 & 1 & $\mathrm{X}$ & $\mathrm{X}$ & $\mathrm{X}$ & $\mathrm{X}$ & $\mathrm{X}$ & $\mathrm{X}$ & $\bar{X}$ & $\bar{X}$ & $\bar{X}$ & $\mathrm{X}$ & $400 \mathrm{~h}$ & $7 \mathrm{FFh}$ & 1024 \\
\hline Load & 1 & $\mathrm{X}$ & $\mathrm{X}$ & $\mathrm{X}$ & $\mathrm{X}$ & $\mathrm{X}$ & $\mathrm{X}$ & $\mathrm{X}$ & $\mathrm{X}$ & $\mathrm{X}$ & $\mathrm{X}$ & $\mathrm{X}$ & $800 \mathrm{~h}$ & FFFh & 2048 \\
\hline Unused & 0 & 0 & 0 & 0 & 0 & 0 & 0 & - & - & - & - & - & $004 \mathrm{~h}$ & 01Fh & 32 \\
\hline
\end{tabular}




\subsection{Message Priorities}

Suggestion for dynamic priority assignment on CAN bus using reserved bits in the CAN protocol [31] have been published. However, it requires modification of CAN protocol and it is not feasible in the current architecture. Instead, first four bits in the CAN identifiers are assigned for message priority. Since 'zero' has higher priority in CAN protocol, all zero bits provide highest priority to that message. Priority field is four bits so there are 16 priorities available for messages. Table 5.3 gives priorities assigned for different types of messages.

Table 5.3: Message priorities

\begin{tabular}{|c|l|c|}
\hline Message type & Messages & Priority \\
\hline Error messages & Error, Pause, Quit, Halt, Reset & 0 \\
\hline Reconfiguration messages & Inform, Enquiry, Power demand, Power reply & 1 \\
\hline Wave message & Inform wave color and d-value & 2 \\
\hline Implementation messages & $\begin{array}{l}\text { Parameter set, Inform and Enquiry of measure- } \\
\text { ments }\end{array}$ & 3 \\
\hline Maintenance & Link delete, new link & 8 \\
\hline Start up & Birth message, Resume, Resume allow & 14 \\
\hline Other messages & Status & 15 \\
\hline
\end{tabular}

\subsection{State Diagram Description}

Each device has six states defined as "Power On", "Device Initialization", "Process Initialization", "Preoperational", "Operational" and "Stopped". The state transition is driven by an internal event or a command issued by command and control center [17]. Figure 5.3 shows the state transitions diagram.

Power on : A device is in this state when powered on or a reset command is issued by command and control center. This state is hardware initialization state, i.e., executing bootstrap loader, loading Special Function Registers (SFR) settings, etc. In this state, the local 
device starts the hardware controller, directory process, communication interface, and CAN controller.

Device initialization : When in this state a directory process starts and sends birth message on the network and waits for Allow Resume message from the command and control center. In this state the device informs that it is turned on and reports its Device ID, thus

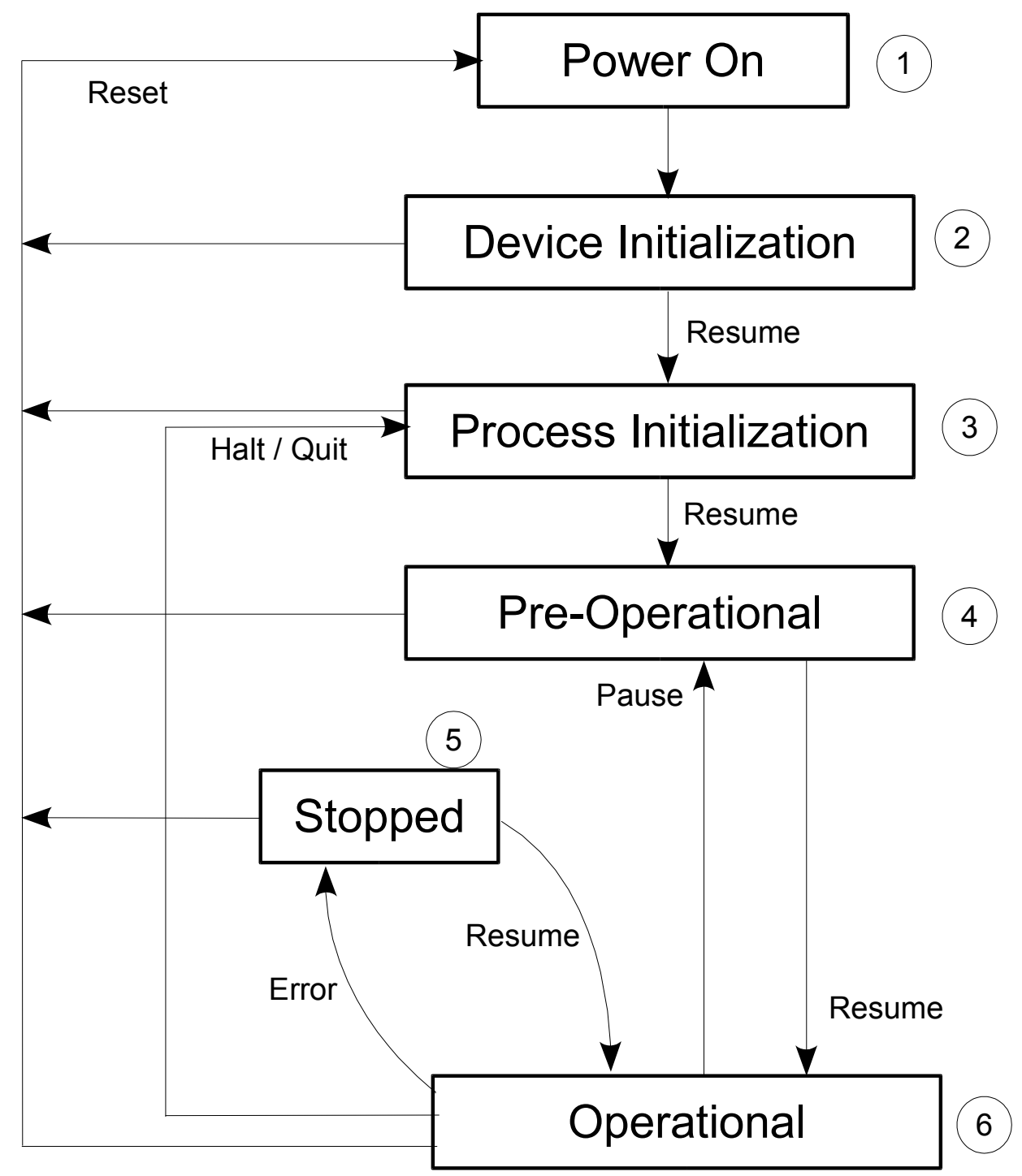

Figure 5.3: Communication and device state diagram 
CCC can verify the address and can inform the user or some other agents, if required. Once the device receive the message it enters the process initialization state.

Process initialization : All process initialization functions are completed in this state. Directory process starts all other processes one by one. In case of RA, the DP informs agent name(s) or number(s). Each process sends a Birth message to inform the command and control center of the agent name through birth message. Command and control center verifies the information, performs sanity check, and sends Allow Resume message.

Preoperational : Tasks related to object dictionary and directory process are carried out in this state. Command and control canter issues commands to update or add new entries. RA inquires about neighbors, their Device IDs, links, and their directions, capacities, etc. Thus, agent learns about its location in the network graph.

Operational: In this state every device actively participates in all communication and performs the operations designated to it.

Stopped: Device/Process enters this state if an error or temporary fault condition occurs in the process, e.g., over temperature. A device/process enters operational state when it detects that the fault is removed or when it receives allow resume message.

Figure 5.4 shows different messages exchanged between command and control center and a device at initialization. Each device sends the birth message with the agent's name. Thus CCC knows that the device is active and then it verifies the address. After verifying the address it sends resume allow message. Then the directory process on the device starts each agent on the device and the agent reports to control center about its process ID. Once all agents start, directory process requests to resume normal operation and waits for CCC response. Control center records all the agents and their respective Process IDs and once again sends resume 
allow message. This final message starts all agents to their normal execution mode, i.e., agents start executing the algorithms.

\subsection{Conclusion}

This chapter presents complete communication structure for agent based SPS reconfiguration system. All agents use these messages to communicate with each other and with command and control center. The unified scheme also considers implementation agents and it is easily possible to extend this messaging scheme to other device that may be present in the system. The use of CAN bus puts restrictions of the amount of data transfer but still the structure is made compatible with CANOpen data structure and FIPA messaging scheme. The communication structure also specifies some network management procedures like error reporting, status messages, and system startup procedure. 


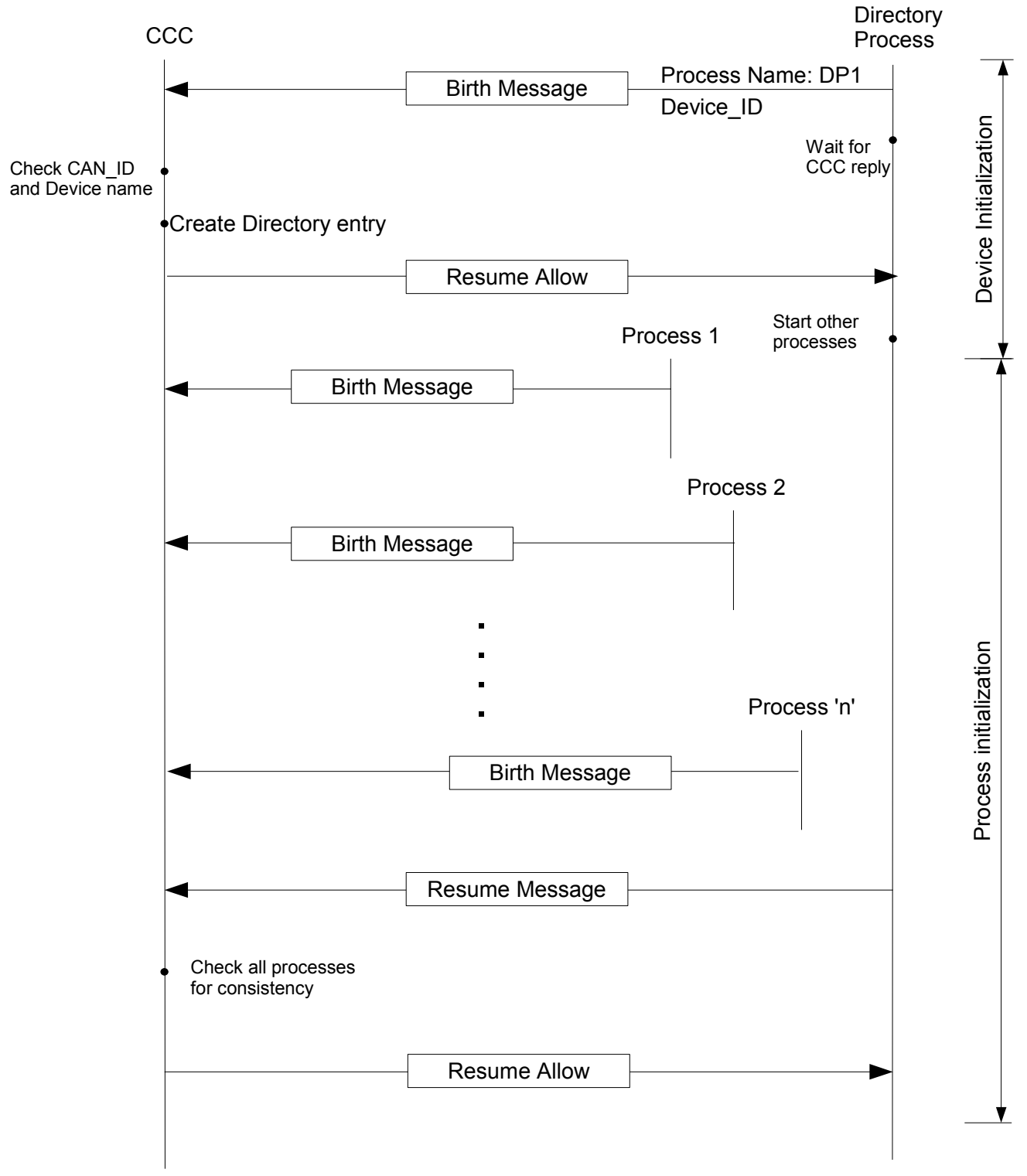

Figure 5.4: Messages exchanged during device start up 


\section{Chapter 6}

\section{System Integration}

The last three chapters explained the agent algorithms and communication architecture. This chapter presents integration of reconfiguration algorithm, termination detection algorithm, agent communication, and power system model. The complete test system consists of four personal computers (Figure 6.1), two computers running reconfiguration agents, one computer as command and control center, and the fourth for power system model. Reconfiguration agents and command and control center communicate over CAN bus using CAN-USB interface. These machines use the communication structure designed in Chapter 5. Power system model in Simulink runs on the fourth machine interfaced with control center using Ethernet through ODBC database channel. Implementation agents are part of the Simulink model and send error codes and system changes to the control center. Control center and Simulink model share a common database. This database contains control settings for Simulink blocks. The user can change load demand and priority or simulate a fault condition. Command and control center detects the changes and relays these to reconfiguration agents. Then the reconfiguration agents solve the reconfiguration problem and send the control settings to control center for implementation in the power system model.

CAN has been chosen due to its suitability for embedded system and flexibility in testing 


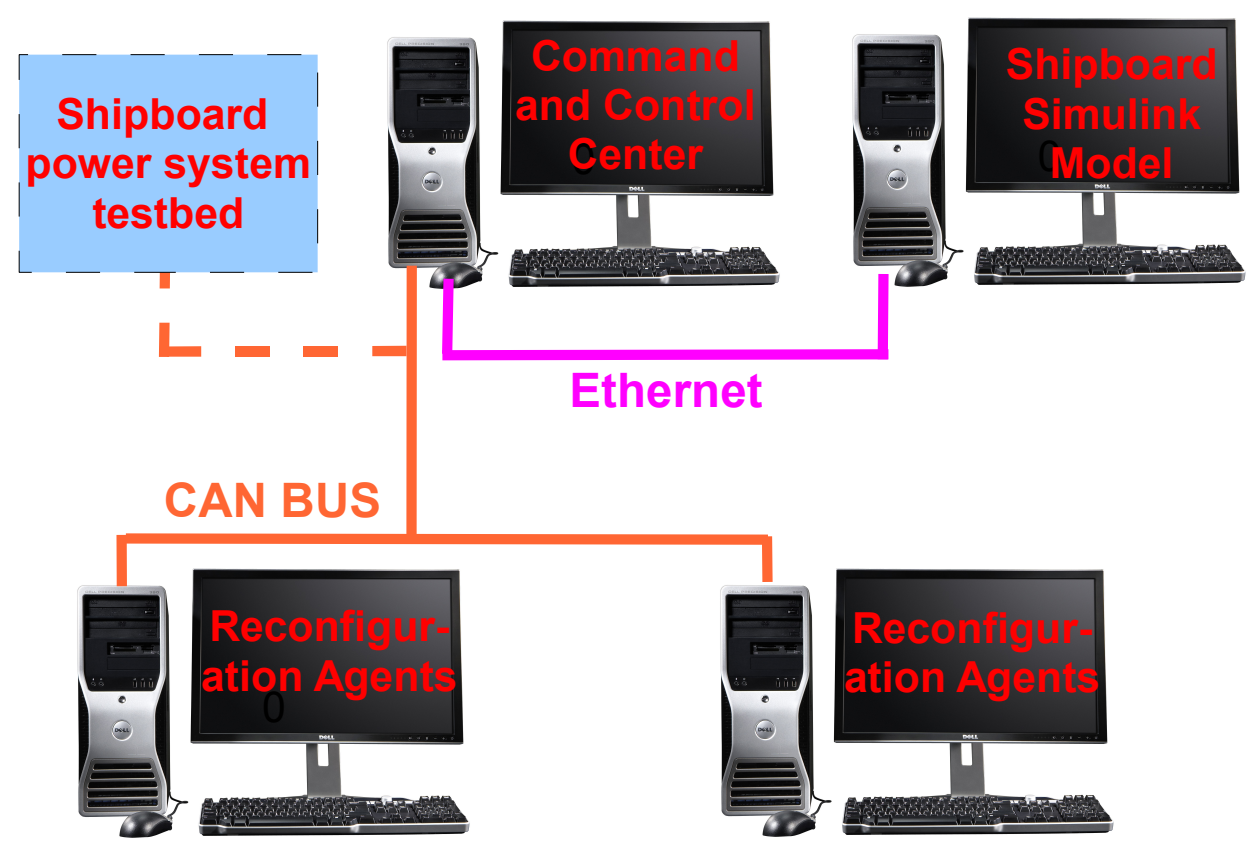

Figure 6.1: Integration of complete system including reconfiguration agents, power system model and command and control center.

different communication and protocol schemes including peer-to-peer and broadcasting. Implementation on ships would require consideration of back-up communication and bus fault recovery. These issues are ignored here. Figure 6.2 shows the layered architecture of this agent based reconfiguration system. All the power system components (power electronics and electromechanical devices) form the physical layer of the system. These devices provide measurements to implementation agents and receive control settings from implementations agents. These devices connect only to respective implementation agents and do not have any direct access to a communication channel. Implementation agents are responsible for reporting changes in power system, faults, or load demand, etc., to reconfiguration agents or command and control center. Each implementation agent can talk to respective reconfiguration agent besides relaying changes in the system, it can decode messages and control the power system component as instructed by the reconfiguration agent. In this integrated test system, Simulink model implements the bottom two layers in soft real-time. The implementation agents communicate through a database shared with control center and control center translates it to 
CAN messages for reconfiguration agents. The next higher layer is the reconfiguration layer. It consists of agents with reconfiguration and termination detection algorithm. These agents can talk to each other and to the command and control center. The last and uppermost layer is the command and control center. It monitors the system, records any faults, errors, changes in system conditions, communication channels, provides an interface between CAN bus and ODBC database, and has a user interface to display the entire system.

The communication medium for agents is CAN bus as it is a common type of fieldbus and drivers are commonly available with microcontroller units and it is easy to use communication medium for embedded systems. A project currently in progress builds a Shipboard power system test bed using microcontrollers that utilize CAN bus. Once completed, the same communication medium can be used for a proof-of-the-concept implementation of multiagent reconfiguration system using hardware model. The Simulink model provides the simulation of the power system under consideration and it is interfaced with reconfiguration system via Ethernet, which is more flexible, and ODBC drivers readily provide data sharing between two computers. The following sections explain implementation of power system model, reconfiguration agents, and command and control center.

\subsection{Power system model}

Figure 6.3 shows the model of shipboard power system built in Simulink. Power system components are represented by first and second order discrete transfer functions to model input-output relationships concerning power and voltage [45]. The Simulink blocks for each device provide user control to change load demand or to simulate a fault, for example, short circuit, loss of supply, etc. This represents the physical layer of the energy management system. 


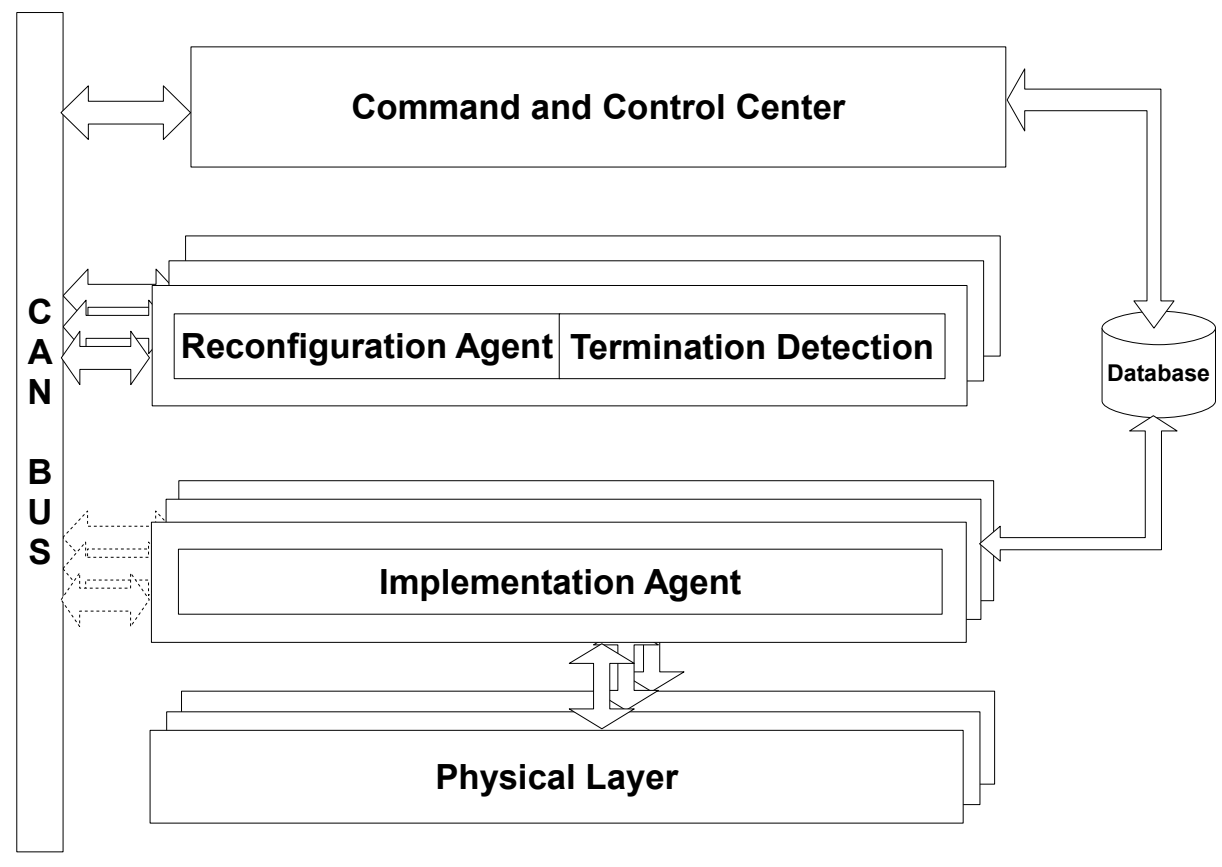

Figure 6.2: Layered architecture

\subsection{Implementaton agent}

The implementation agents represent the controllers for the power system devices. These devices are also capable of providing measurements, fault conditions or change in system parameters like load demand. The implementation agent tasks and communication are simulated by creating a common database between Simulink model and control center. This database contains Simulink model control variables like power reference, controller settings, on/off signal and user-controlled variables like power demand, fault type, etc. Command and control center detects these changes in the database and relays them to the appropriate reconfiguration agent. For the reverse path, reconfiguration agents send the control settings to CCC and then CCC translates these messages into database quantities to be by the Simulink blocks. 


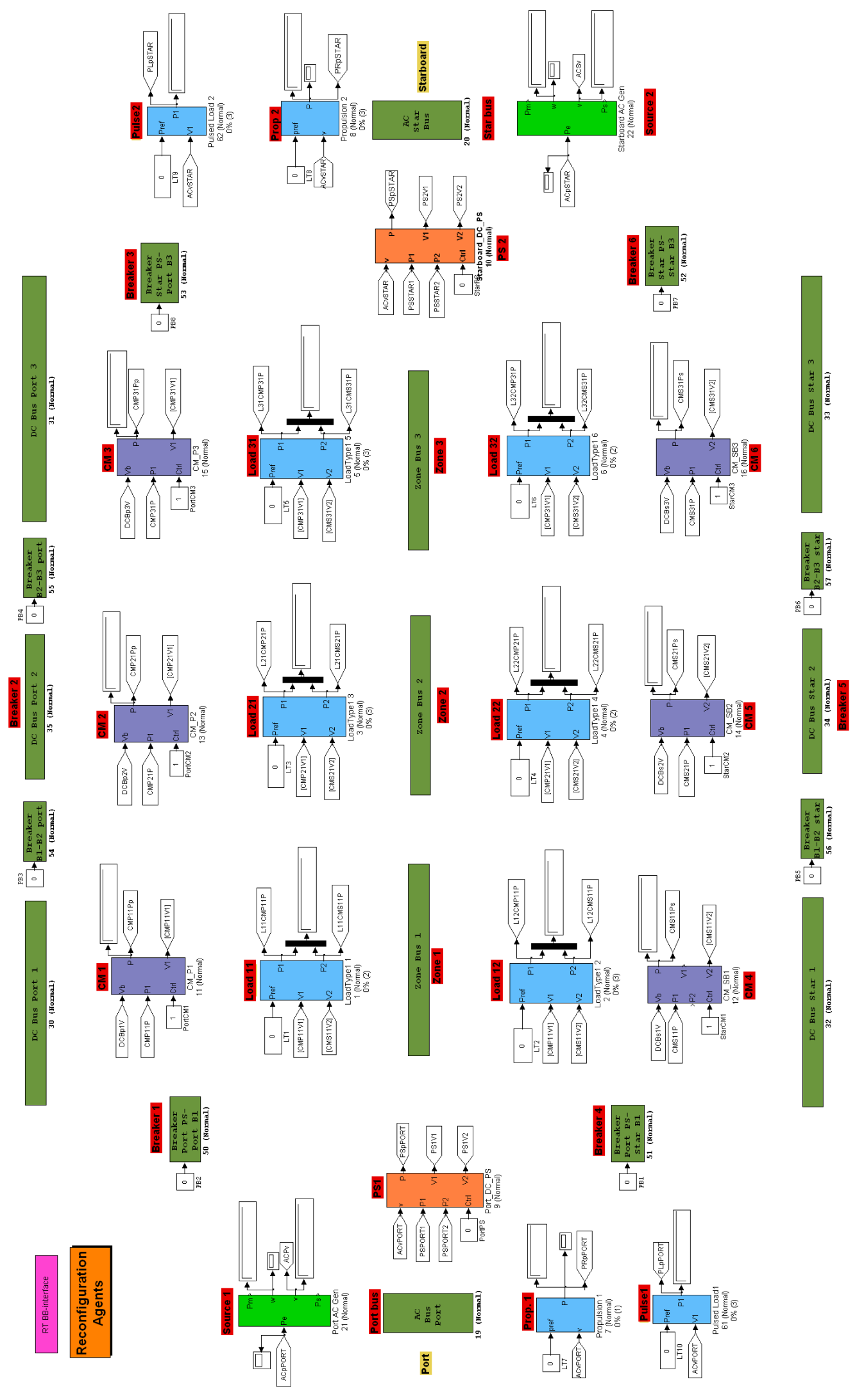

Figure 6.3: Power system model 


\subsection{Reconfiguration agents}

The shipboard power system shown in Figure 3.1 is modeled as directed graph where a reconfiguration agent (Figure 3.3) represents each node. This model requires 31 reconfiguration agents and 34 links. These agents are randomly divided between two PCs, with one machine running 15 agents and other machine running 16 agents. The reconfiguration algorithm and termination detection algorithm is implemented in Matlab with each agent represented as a Matlab object. These agents communicate with each other and with the command and control center using CAN bus and follow communication structure and messages defined in Chapter 5.

\subsection{Command and Control Center}

As mentioned before, CCC displays overall system status, allows user to control agents, and acts as a translator between CAN messages and Simulink ODBC-interface. Each agent updates the information every time it finishes a reconfiguration. CCC displays a system graph with agent information, current configuration, overall system data including CAN addresses of all the agents, link capacities, load demands, maximum generation capacity, and system faults. The reconfiguration agent information includes type, parent, d-value, incoming links, outgoing links, power flow, and residue on these links. The data is useful for diagnosis, collecting statistics, and analyzing performance of reconfiguration agents. To test the reconfiguration algorithm under fault conditions, CCC provides control over the reconfiguration agents. It allows the user to change demand, priority of a load agent, and maximum capacity of a link. It also allows users to deactivate links or agents. Figure 6.4 shows a screen shot of the user interface for the command and control center. 


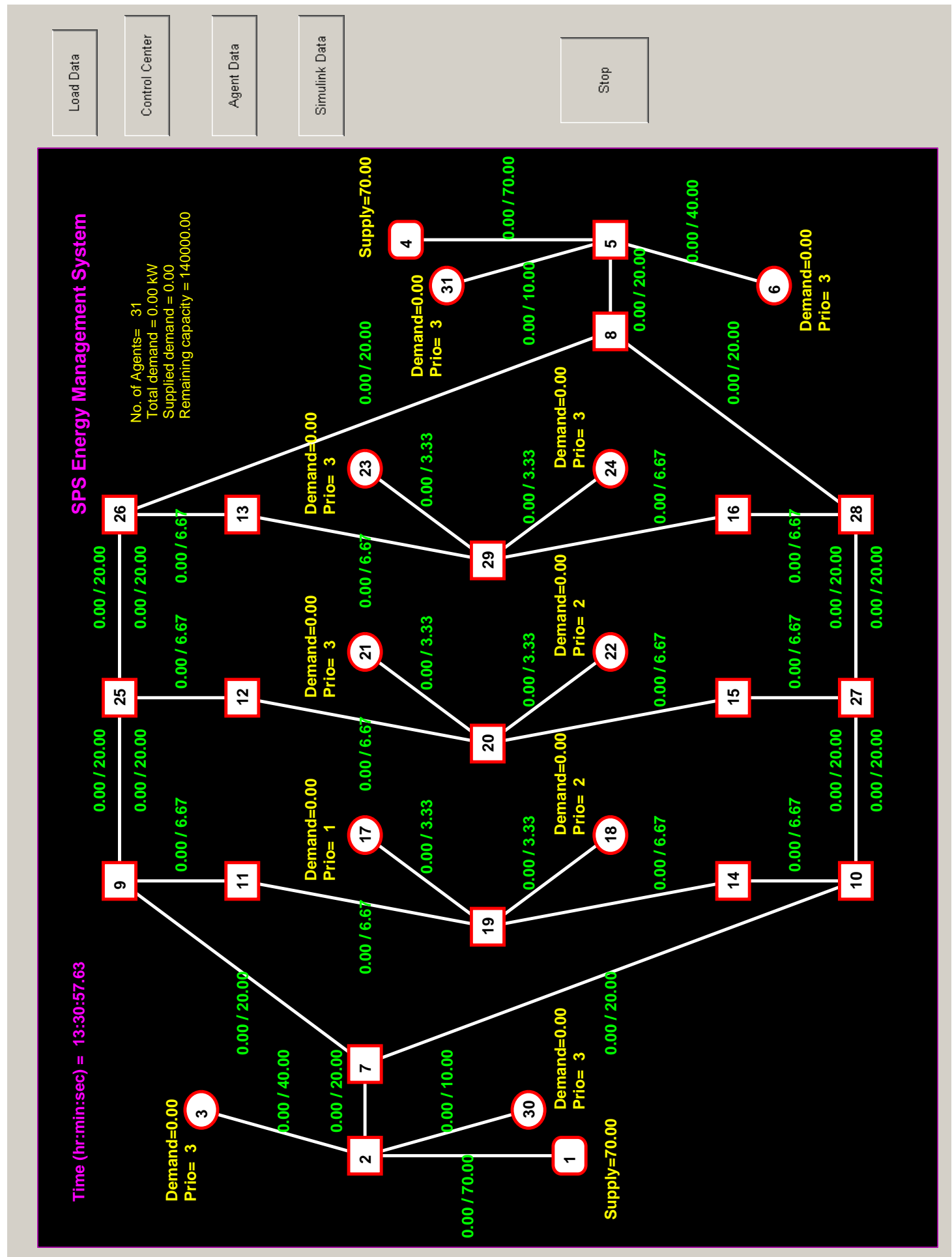

Figure 6.4: Command and control center - Graphical user interface 


\section{Chapter 7}

\section{Testing and results}

The designed reconfiguration system has been tested for different scenarios, e.g., initial startup with and without full load, changes in load demand, changes in line capacity, increasing the demand beyond generation capacity, loss of line, etc. Following sections discuss some of these test cases. The reconfiguration algorithm and termination detection algorithm is developed in Matlab and agents are executed in random sequence. The testing includes two different setups, in the first case, all agents are located on a single computer, and in the second case agents are distributed on multiple computers. Statistical information collected while running the test scenarios a thousand times each is used in analyzing the performance.

\subsection{Initialization}

In the 'Initialization' study, all agents start from their default values and negotiate to form spanning forest by breadth first search algorithm, store the parent and children list, and update d-values as the shortest distance from the root. The load demand is set to zero in the initialization scenario. The purpose of this test is to analyze the messages and time required to form the basic tree structures in the network. The trees formed in the initialization remain 


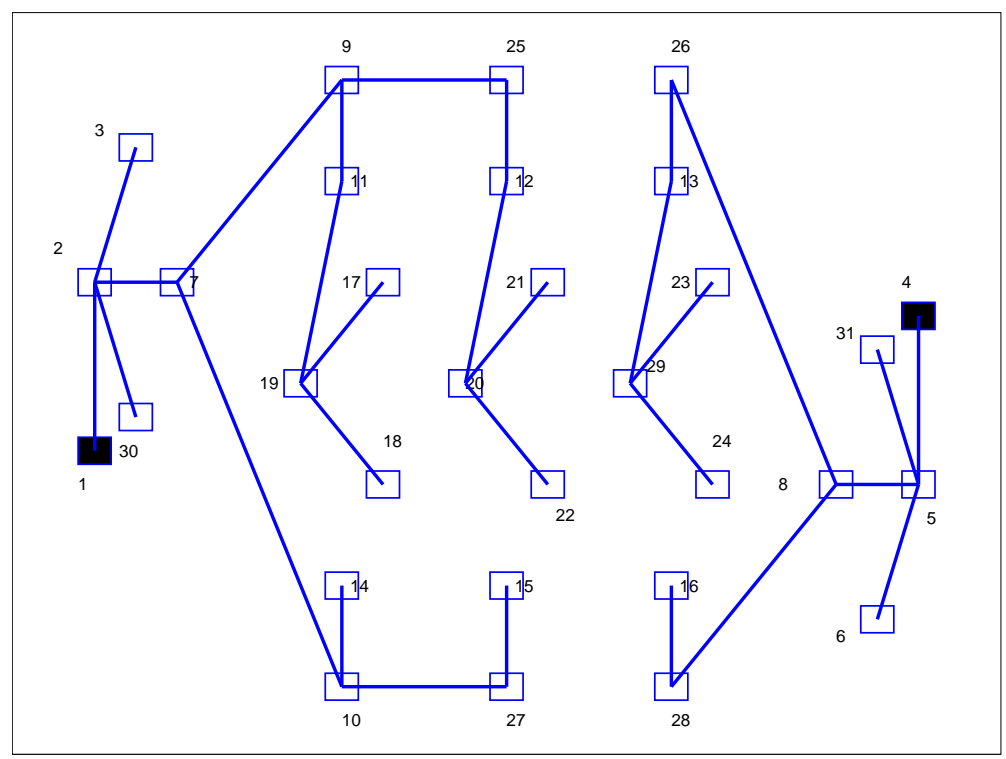

Figure 7.1: Spanning forest with two tree structures, source agents 1 and 4 are the trees' root nodes.

intact unless there is some change in the physical layout of the power system, e.g., removing a line. Figure 7.1 shows an example of the two spanning trees formed by the agents. The source agents 1 and 4 are roots of the two trees.

\subsection{System startup at full load}

This test scenario is similar to the initialization case except demand of all loads is set to 100\%. All agents start from their default values, form the tree structure, and then negotiate to solve the power flow to supply load demand. Figure 7.2 shows one possible solution to the reconfiguration problem. Figure 7.4(a) shows the number of reconfiguration moves made by agents during the execution period. Figure 7.4(b) shows total time required for the execution. Figure 7.4(c) gives the time required to detect the termination by all agents after they finish the reconfiguration algorithm. This termination detection time is the extra time required by the wave algorithm to detect the termination as compared to the blackboard approach. Figure 7.5(a) gives the comparison of control messages and reconfiguration messages. The 


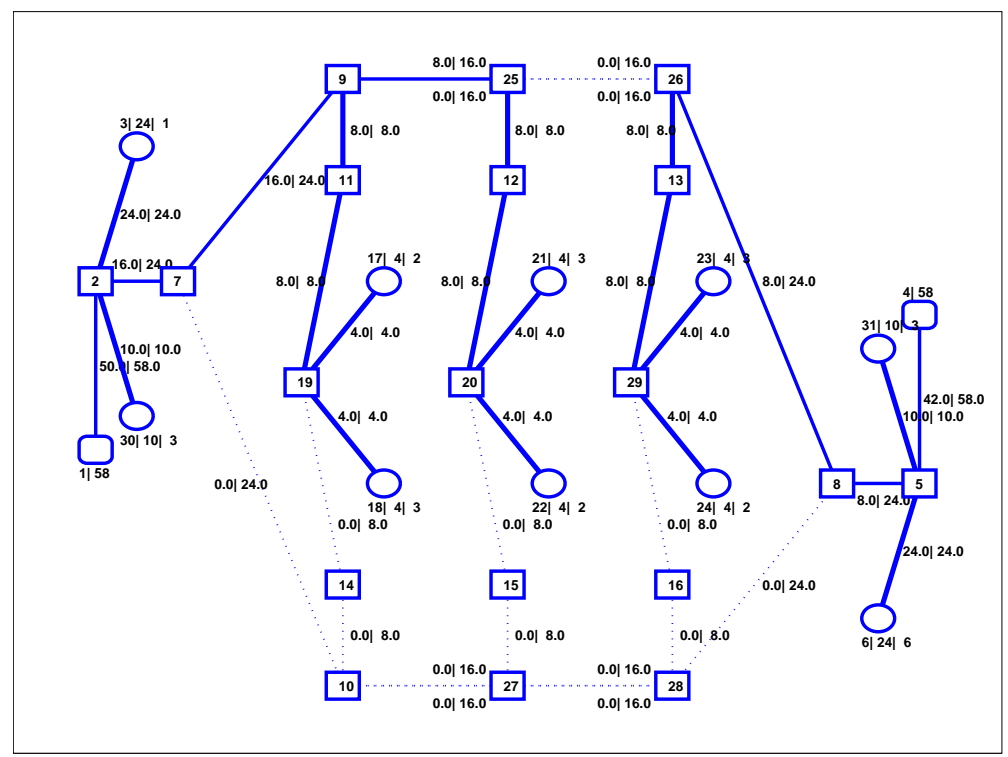

Figure 7.2: One possible solution to reconfiguration problem for startup case. Link information: flow | Capacity. Load agent information: Agent number | demand | Priority. Source agent information: Agent number | Capacity

comparison of this figure with Figure 7.5(b) reveals that the number of control messages is almost same. This similarity signifies that the reconfiguration algorithm and messages have
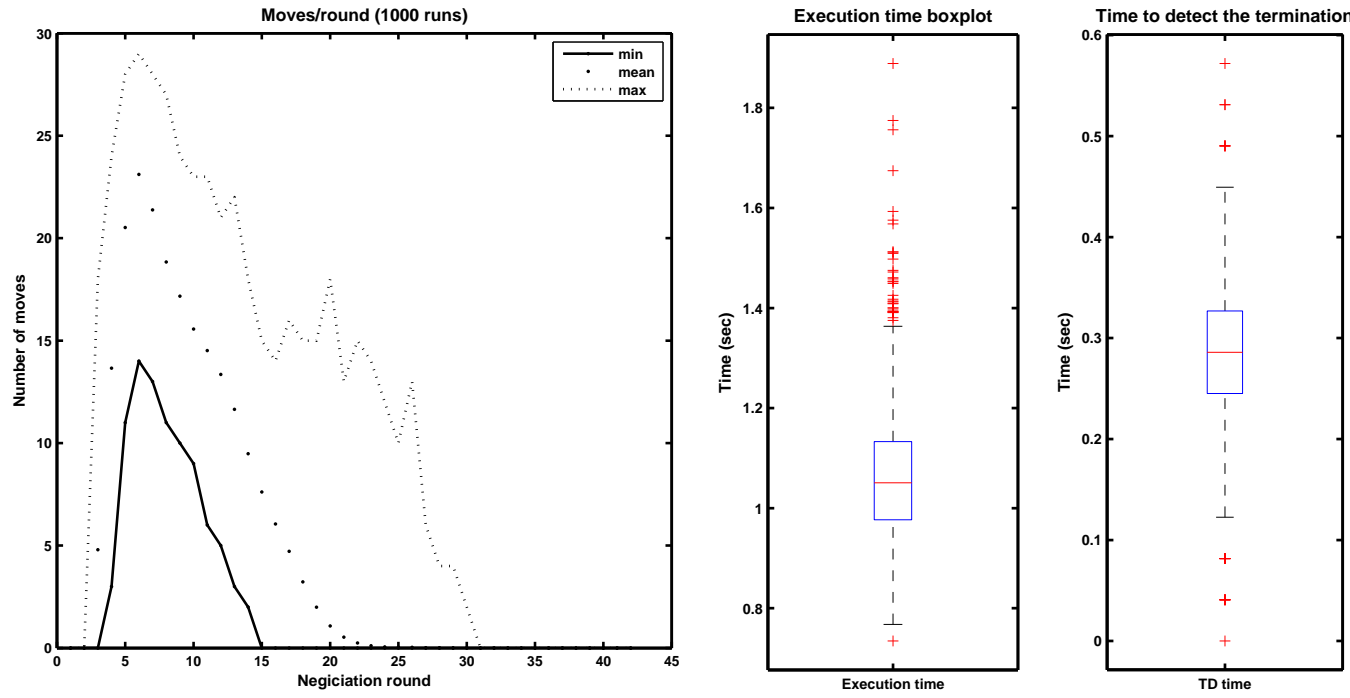

(a) Minimum, Mean and Maximum number of moves made by (b) Box plot of execution (c) Box plot for termination all agents during the execution. time detection time(TD)

Figure 7.3: Timing analysis for startup case 


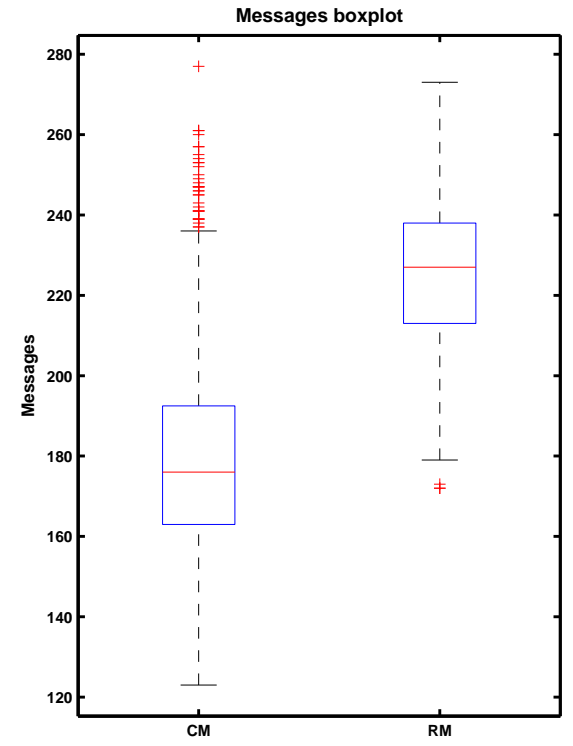

(a) Messages for initialization case

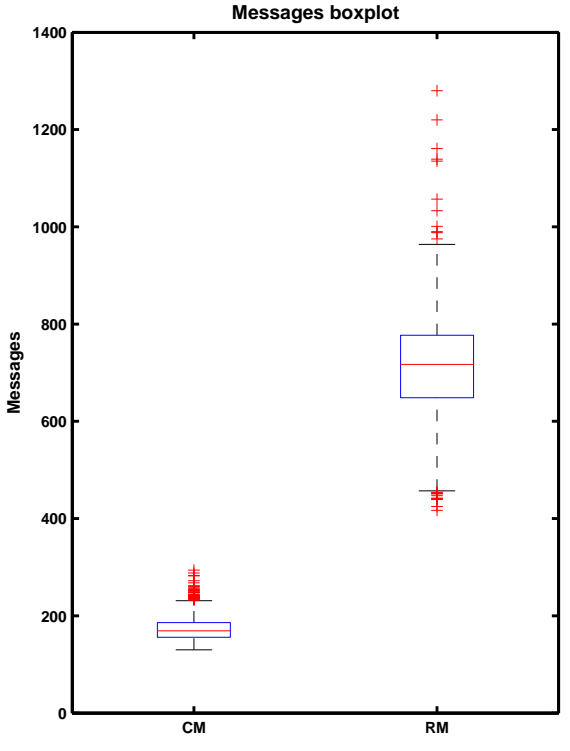

(b) Messages for startup case

Figure 7.4: Box plots for messages exchanged between agents. (CM: Control messages, RM: Reconfiguration messages)

higher priority over wave messages of the termination detection algorithm. The termination detection algorithm does not interfere with the execution of reconfiguration algorithm.

Boxplot [25]: It is a convenient way of graphically depicting the five-number summary, which consists of the smallest observation, lower quartile, median, upper quartile, maximum observation and the interquartile range (IQR), i.e., first quartile to the third quartile. The horizontal line represents the median of the data set and the box spans the interquartile range. Interquartile range is the range of values within which middle $50 \%$ of the ranked data are found. The vertical line is called as whisker and it spans data values of 1.5 times the box height (or IQR) on each side of the box. Any data points outside the whiskers are extremes and are represented by + signs. 


\subsection{Other test cases}

The reconfiguration system is tested for different reconfiguration scenarios to test the performance, number of messages exchanged, execution time, and solution correctness. An automated code tests the solutions for any capacity violations, unsupplied load demand, total generator power vs. total load demand, and agent termination status. The test conditions include change in load demand, change in load priority, and change in line capacity. Some very important reconfiguration cases where tree reconfiguration is also required are presented in the remaining of the chapter.

In the first case the system considered is operating as shown in Figure 7.2 and the power line from node two to seven is disconnected. This removal forces the generator agent four to supply all the zonal loads. The change in power system structure also changes the spanning trees. One possible tree structure is shown in Figure 7.6(a) and reconfigured power system is shown in Figure 7.6(b). Figure 7.6 shows statistical results for this test scenario.

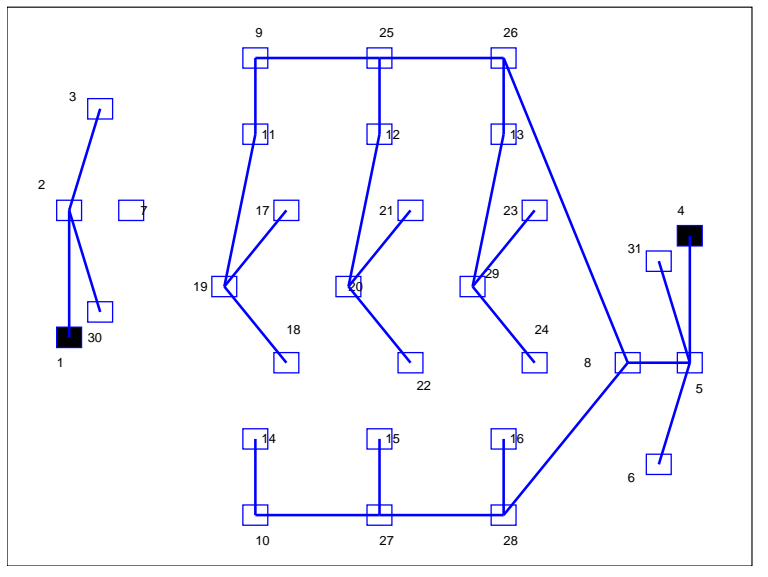

(a) Tree structure

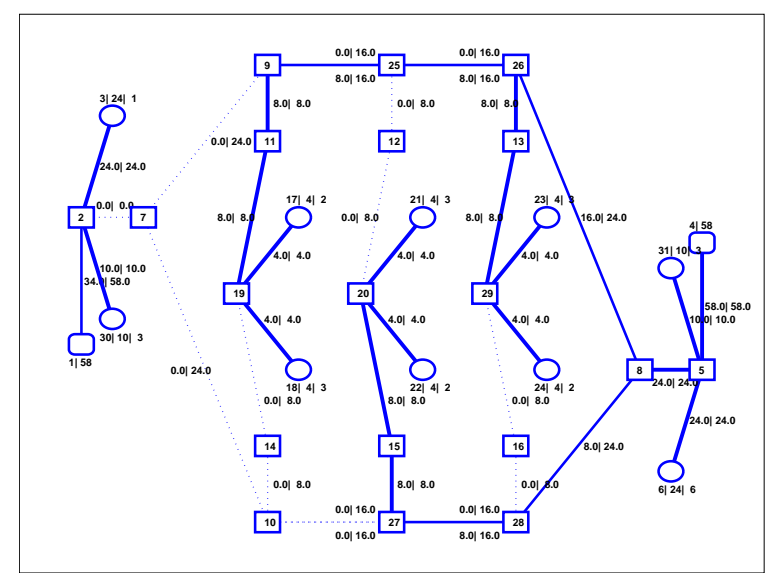

(b) Reconfiguration solution

Figure 7.5: Trees and power flow solution for case: line 2-7 is disconnected.

In the next test case two faults are simultaneously introduced to the system operating in conditions shown in Figure 7.2: (i) line 2-7 is disconnected and (ii) capacity of line 5-8 is reduced to $50 \%$. The tree structure is the same as in the previous case (Figure 7.6(a)) but 


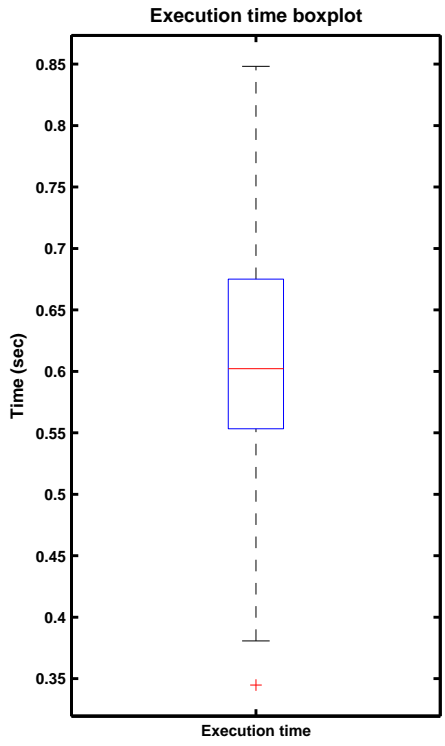

(a) Execution time

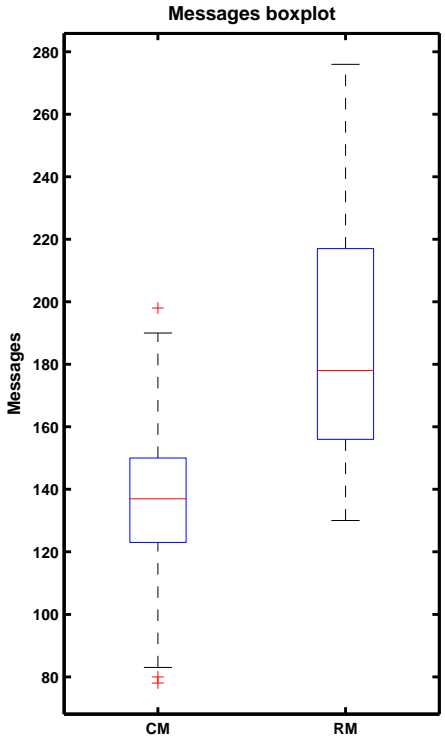

(b) Messages

Figure 7.6: Test results for case: line 2-7 is disconnected.

the low priority loads have to be dropped and capacity of line 5-8 is fully utilized to supply remaining high priority zonal loads. The reconfiguration solution is shown in Figure 7.7 with statistical results in Figure 7.9.

The test performed with disconnecting a line shows that the system is capable of handling extreme events. The loss of line 2-7 separates the power system in two parts and still the agent based system is capable of reconfiguring the system and able to terminate successfully. This result shows the agents survivability, i.e., even if the system separates in two distinct parts, the parts can successfully reconfigure the system. The test results for number of messages and time required for reconfiguration does not change when there is enough line capacity available to supply all the load demand. When there is less line capacity or generation than load demand, the reconfiguration time is approximately three times the normal reconfiguration time and the reconfiguration finishes in less than three seconds. 


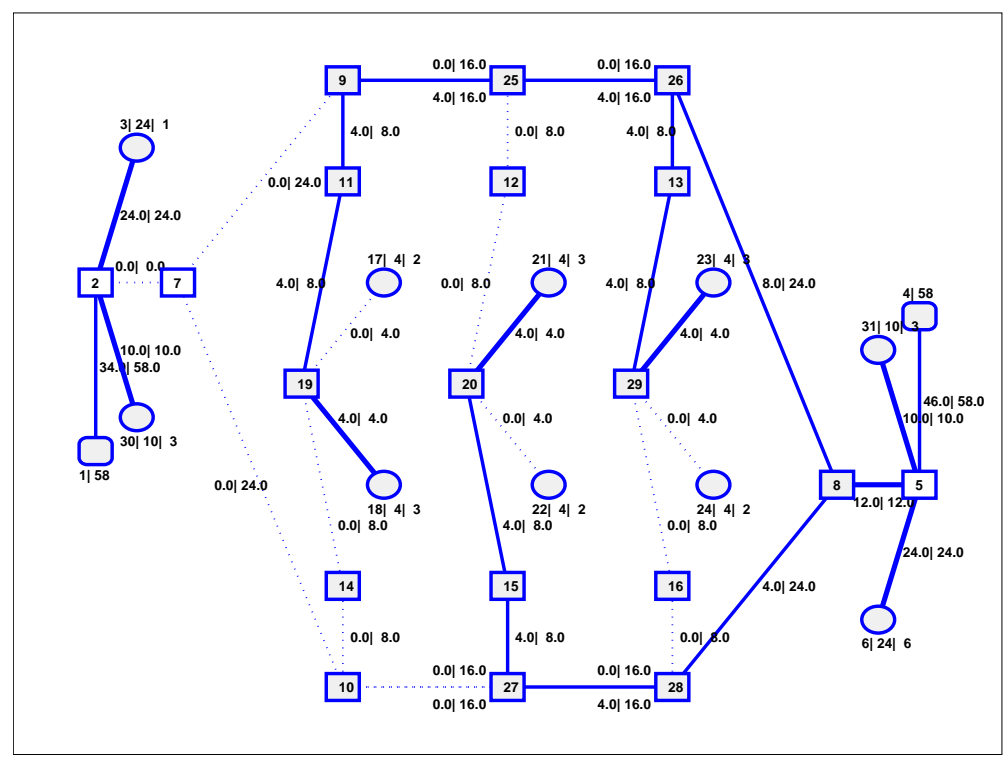

Figure 7.7: Reconfiguration solution for case: line 2-7 is disconnected and capacity of line 5-8 $=50 \%$.

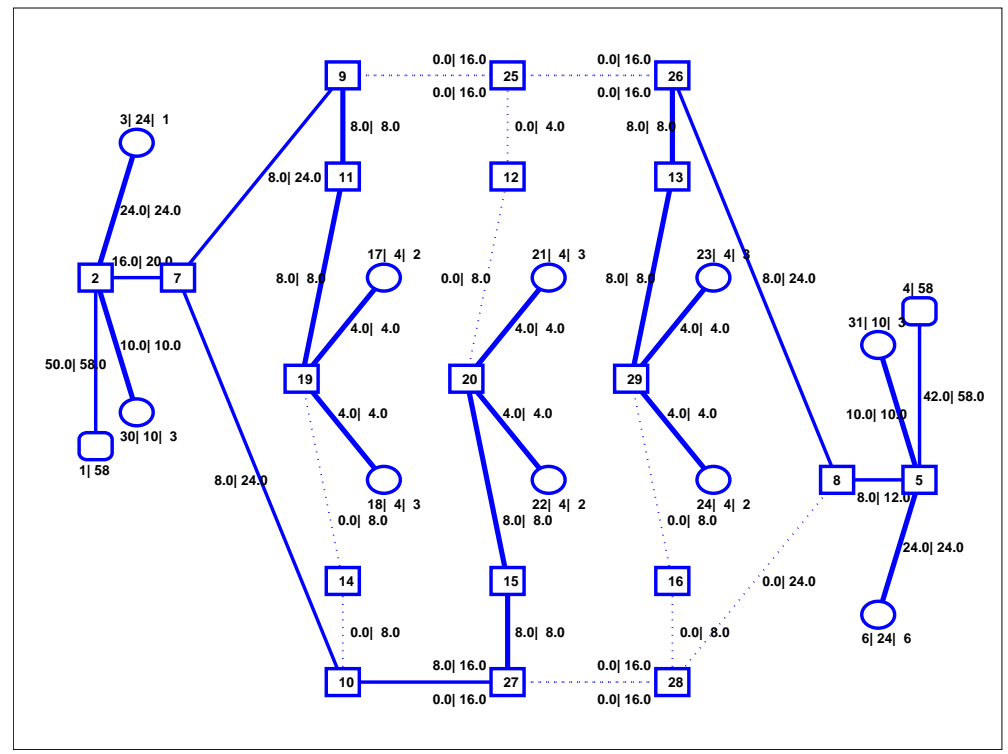

Figure 7.8: Test results for case: line 2-7 is restored to full capacity and capacity of line 5-8 $=50 \%$. 
Figure 7.8 shows the reconfiguration solution when link 2-7 is restored back to full capacity. When the link is brought back into service all the loads get supplied and system returns to normal operation.

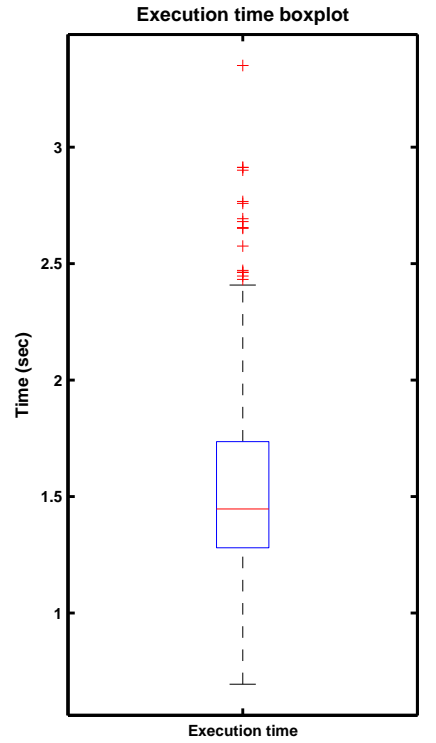

(a) Execution time

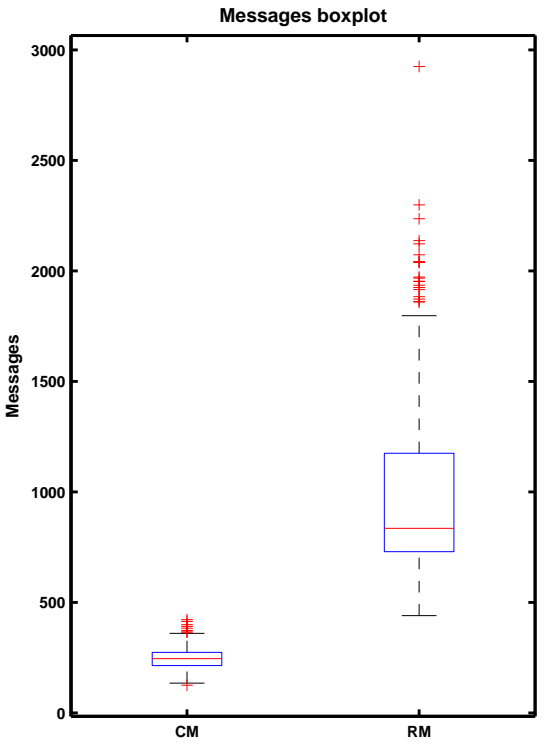

(b) Messages

Figure 7.9: Test results for case: line 2-7 is disconnected and capacity of line $5-8=50 \%$.

The next comparison shows the execution time and messages required for startup case with four different agent execution methods. In random execution, the agents are executed in random sequence but in the equal probability execution, each agent is executed for equal number of times within a finite duration. In the third method, the 31 agents are distributed on two different machines and CAN bus communication is established between them. In the fourth case the agents are distributed on three different machines with CAN bus communication. Figure 7.10 shows the execution time and Figure 7.11 shows messages exchanged. In random execution, the number of messages is almost same and the execution time is only 1.5 times larger than equal probability execution. Therefore, in completely distributed system with each agent running on separate microcontroller device, it will take more time to execute but it will not increase the information exchanged. 


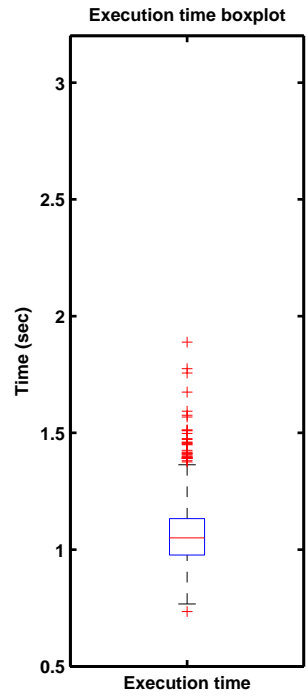

(a) Equal probability

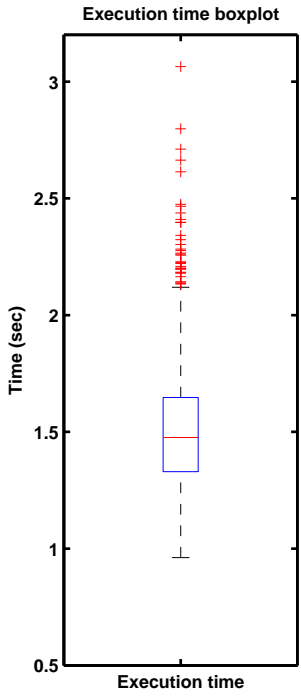

(b) Random
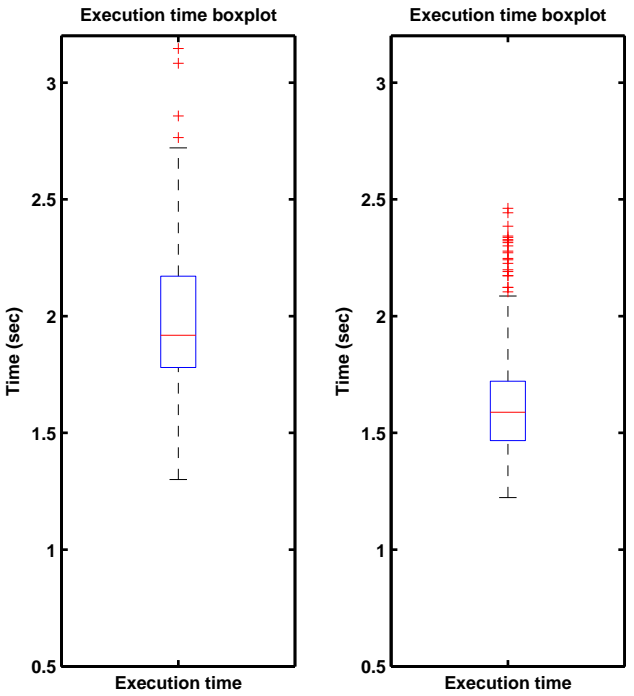

(c) 2 computers with com- (d) 3 computers with communication munication

Figure 7.10: Execution Time

When agents are distributed on two different machines the execution time (Figure 7.11(c)) increases due to extra processing required while sending the messages. Figure $7.11(\mathrm{~d})$ shows

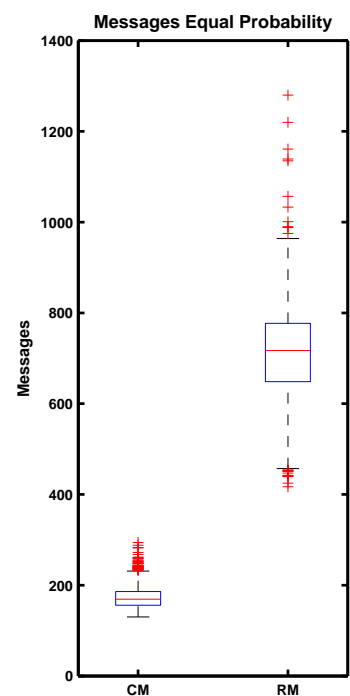

(a) Equal probability

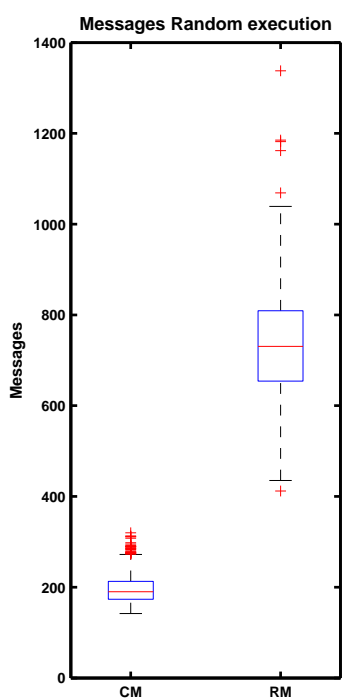

(b) Random

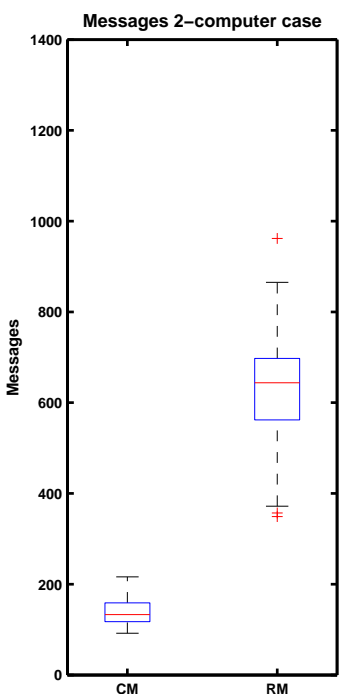

(c) 2 computers with communication

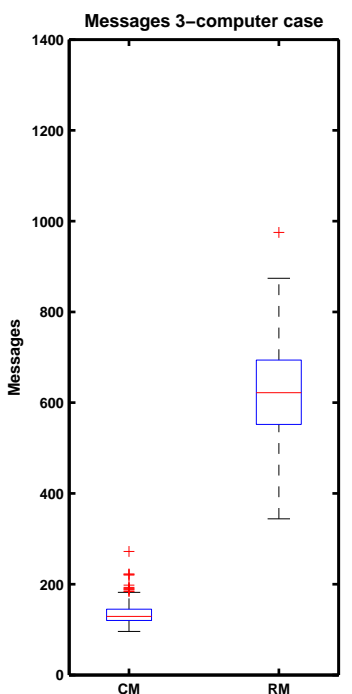

(d) 3 computers with communi-
cation

Figure 7.11: Messages exchanged. CM: Control messages, RM: Reconfiguration Messages. 
the effect of parallel processing on execution time. The distribution of agents on three machines reduces execution time. The messages exchanged in all the four cases are almost equal, so it can be concluded that distributed execution of the agents does not change the amount of information exchanged between agents. 


\section{Chapter 8}

\section{Conclusion}

A new agent based reconfiguration algorithm with termination detection is presented. The simulation is performed on multiple machines with CAN bus communication. The method uses local computations and communication with neighbors to reconfigure the power system. The complete system includes power system model, a control center and the reconfiguration agents. This system operation is completely automatic; the agents respond to any fault in the power system, reconfigure the system, find a new power flow and then implement it in the power system. The entire operation happens without any user intervention. This autonomous scheme becomes possible due a newly designed termination detection algorithm and communication structure developed for this specific application. The termination detection algorithm acts as a supplementary program and does not interfere with the reconfiguration algorithm. The promising result of this work is the ability of all the agents to work independently without any central control or database. Experimental results show that, when the multiagent system divides into two parts due to a line outage, both parts can work independently and reconfigure the system. When the fault is removed, the agents detect the change, adjust their parameters and again start working as one system. The reconfiguration algorithm considers bidirectional flow by using two back to back uni-directional links. It also provides the option to receive power 
from only one source or to receive power from multiple sources simultaneously. The algorithm effectively responds to changes in the loads' priorities and system changes and reconfigures the system to supply high priority loads. This work is a step towards practical implementation of energy management system with distributed microcontroller units situated close to power system components and communication to reconfigure the power system without any central control.

\subsection{Future work}

In this multiagent system, load priorities can be changed only by the users, i.e., the load agents do not change priority by themselves. There is a potential improvement possible for changing load priorities. Load agent can be allowed to change its priority by negotiating with other load agents based on its category and mission condition. This change will provide more flexibility in reconfiguration when there is less available power than demand. The agent algorithm comprises reconfiguration and termination detection algorithms. Once the agent detects termination, it sends control signals to implementation agent. This step may require coordination of control actions among different agents to maintain power system stability and integrity. The coordination can be performed at either implementation layer or reconfiguration layer. Reconfiguration agents can use the wave algorithm with additional rules and perform their actions when a wave visits the agents.

CAN bus used for the communication is a major pitfall of the system. It is used in the system because the CAN controllers are easily available, it is simple to implement and yet it gives low error rates at $1 \mathrm{Mbps}$ data rate at $40 \mathrm{~m}$ cable length. The problem with CAN bus is the bus architecture and fixed termination impedance for proper working. Therefore, if the cable breaks, agents will not be able to communicate. In order to provide a good communication channel, an alternative method such as backups, wireless or Ethernet are necessary. Agent simulation environment is Matlab, which does not support multithreading 
or parallel processing. Even though the simulation is carried out in multi-computer setup, it is limited to three computers only. An interesting future step would be to simulate the agents using Java Agent DEvelopment framework (JADE). JADE simplifies multiagent system development and provides simulation environment using Java engine. Furthermore, to test the agent based system in embedded environment should be considered. CAN communication can also be used in this proof-of-concept testing because the drivers and CAN communication interfaces are easily available with microcontroller units. 


\section{Appendix A}

\section{Error Codes}

Following list gives predefined error messages used by reconfiguration agents and implementations agents. The hexadecimal codes are compatible with the error codes specified in CANOpen standard.

PreDefinedErrorMsg $=$

0x0002 Flow greater than capacity.

0x0003 Distance greater than maximum value.

0x0010 Out of memory.

0x0011 Object not found in object dictionary

0x0012 Invalid parameter.

0x0013 Operation time out.

0x0014 Parameter out of range.

0x0015 Invalid Process.

0x0016 Short message length.

0x0017 Message not understood.

0x0030 Over voltage.

0x0031 Over temperature. 
0x0032 Over current (short circuit).

0x0033 No input (measurement device).

0x34zz Input range exceeded on channel zz.

0x0036 No sensor input.

0x0037 Unstable control.

0x0038 Output saturation.

0x0039 Imbalanced load.

0x004a Overheating.

0x004c On standby supply.

0x004e No actuator/ implementation agent response. 


\section{References}

[1] Foundation for Intelligent Physical Agents, website http://www.fipa.org.

[2] S \& C Electric Company, website http://www.sandc.com.

[3] Lecture Notes in Computer Science. Springer Berlin / Heidelberg, 2001, vol. 2118/2001, ch. The Evolution of contract net protocol, p. 257.

[4] Agentlink, "Chilled water system automation, Rockwell Automation - A case study, case study," Dec 2005.

[5] IEEE Standard for Binary Floating Point Arithmetic, ANSI/IEEE Std 754-1985, Aug 1985.

[6] M. Arias-Albornoz and H. Sanhueza-Hardy, "Distributed network configuration for minimum energy supply cost," IEEE transactions on Power System, vol. 19, no. 1, Feb 2004.

[7] F. Bellifemine, A. Poggi, and G. Rimassa, "Developing multiagent systems with FIPAcompliant agent framework," Software: Practice and Experience (SP\&E), vol. 31, no. 2, 2001.

[8] CAN Specification Version 2.0, Bosch GmbH, 1991.

[9] G. Bracha and S. Toueg, "Resilient consensus protocols," PODC '83: Proceedings of the second annual ACM symposium on Principles of distributed computing, ACM Press, pp. $12-26,1983$. 
[10] K. L. Butler-Purry and N. D. R. Sarma, "Intelligent network reconfiguration of shipboard power system," Power Engineering Society General Meeting, 2003, IEEE, vol. 4, pp. 2435-2439, July 2003.

[11] M. Calisti, "Abstracting communication in distributed agent-based systems," Proceedings of Concrete Communication Abstractions of the next Distributed Object Systems, European Conference on Object-Oriented Programming, Workshop ECOOP02, Malanga, Spain, June 2002.

[12] J. Carroll, K. Nagaraj, A. Arapostathis, W. M. Grady, and E. J. Powers, "Dynamic reconfiguration preserving stability," Electric Ship Technologies Symposium, IEEE, pp. 105-107, July 2005.

[13] K. M. Chandy and L. Lamport, "Distributed Snapshots: Determining global states of distributed systems," ACM Transactions on Computer Systems, vol. 3, no. 1, pp. 63-75, Feb 1985.

[14] CANOpen Application layer and communication profile, CiA, Feb 2000, DS 301, Version 4.02 .

[15] Device profile measuring devices and closed loop controllers, CiA, May 2002, DS 404, Version 1.2.

[16] Electronic data sheet specification for CANOpen, CiA, Jan 2005, DS 306, Version 1.1.

[17] CAN application layer for industrial application, DS 201 to 207 Version 1.1, CiA Standard, 1996.

[18] K. Decker, "Coordinating intelligent agents," in Lecture Notes in Computer Science. Springer Berlin / Heidelberg, 2002, vol. 2403/2002. 
[19] K. Etschberger, R. Hofmann, A. Neuner, U. Weissenrieder, and B. Wiulsroed, "A failure -tolerant CANOpen system for marine automation systems," rth International CAN Conference, CiA, vol. Safety, 2000.

[20] A. Feliachi, K. Schoder, S. Ganesh, and H. J. Lai, "Distributed control agents approach to energy management in electric shipboard power system," Power Engineering Society General Meeting, 2006, June 2006.

[21] T. Finin, R. Fritzson, D. McKay, and R. McEntire, "KQML as an agent communication language," Proceedings of the 3rd International Conference on Information and Knowledge Management (CIKM'94), pp. 456 - 463, 1994.

[22] T. Finin, Y. Labrou, and J. Mayfield, Software Agents. Cambridge MA USA: MIT Press, 1997, ch. KQML as an Agent Communication Language, pp. 291-316.

[23] N. Francez, "Distributed termination," ACM Transactions on Programming Language and Systems, vol. 2, no. 1, pp. 42-55, Jan 1980.

[24] N. Francez and R. Michael, "Achieving distributed termination without freezing," IEEE Transactions on Software Engineering, vol. SE-8, no. 3, pp. 287-292, May 1982.

[25] M. Frigge, D. C. Hoaglin, and B. Iglewicz, "Some implementations of boxplot," The American Statisticians, vol. 43, no. 1, pp. 50-54, Feb. 1989.

[26] S. Ganesh, K. Schoder, H. J. Lai, A. Al-Hinai, and A. Feliachi, "Energy management system with automatic reconfiguration for electric shipboard power systems," Proc. of ASNE Reconfiguration and Survivability Symposium, RSS 2005, Feb 2005.

[27] S. B. Ganesh, "Multiagent Autonomous Energy Management," Master Thesis, West Virginia University, 2005. 
[28] S. Ghosh, A. Gupta, and S. V. Pemmaraju, "A self-stabilizing algorithm for the maximum flow problem," Computers and Communications, 1995. Conference Proceedings of the 1995 IEEE Fourteenth Annual International Phoenix Conference on, pp. 8-14, Mar 1995.

[29] C. L. Giles and K. C. Jim, "Learning communication for multiagent systems," Workshop on Radical Agent Concepts, pp. 377-392, 2002.

[30] E. Godard, Y. Metivier, M. Mosbah, and A. Sellami, "Termination detection of distributed algorithms by graph relabelling systems," in International Conference on Graph Transformations 2002 (ICGT). Springer-Verlag Berlin Heidelberg, 2002, pp. 106-119.

[31] S. Hasnaoui and A. Bouallegue, "A proposal modification of CAN protocol to support a dynamic priority policy being able to implemented on CAN fieldbus controller components," Industry Applications Conference, 2000, vol. 2, pp. 1129-1136, 2000.

[32] K. Huang, D. Cartes, and S. Srivastava, "A multiagent based algorithm for meshstructured shipboard power system reconfiguration," Intelligent Systems Application to Power Systems, Proceedings of the 13th International Conference on, Nov 2005.

[33] Y. Huang, "Fast reconfiguration algorithm development for shipboard power systems," Master Thesis, Mississippi State University, 2005.

[34] F. Jacques, Multiagent systems an introduction to distributed artificial intelligence. Addison-Wesley, 1999.

[35] N. R. Jennings, K. Sycara, and M. Wooldridge, "A roadmap of agent research and development," Journal of Autonomous Agents and Multi-Agent Systems, vol. 1, no. 1, pp. 7-38, 1998.

[36] S. Jonnavithula and R. Billinton, "Minimum cost analysis of feeder routing in distributed system planning," IEEE transactions on Power Delivery, vol. 11, no. 4, Oct 1996. 
[37] Y. Labrou, T. Finin, and Y. Peng, "The current landscape of Agent Communication Languages," Intelligent Systems, IEEE Computer Society, vol. 14, no. 2, March/April 1999.

[38] Y. Labrou, Mutli-agents systems and applications. Springer-Verlag New York, Inc., 2001, ch. Standardizing agent communication, pp. 74-97.

[39] N. A. Lynch, Distributed Algorithms. Morgan Kaufmann, 2003.

[40] J. Matocha and T. Camp, "A taxonomy of distributed termination detection algorithms," Journal of System and Software, vol. 43, no. 3, pp. 207-221, 1998.

[41] A. Muhammad, "Graph, simplicial complexes and beyond: Topological tools for multiagent coordination," PhD Thesis, Georgia Institute of Technology, 2005.

[42] R. Olfati-Saber and R. M. Murray, "Agreement problems in networks with directed graphs and switching topology," Decision and Control, 2003. Proceedings. 42nd IEEE Conference on, vol. 4, pp. 4126-4132, Dec 2003.

[43] — - "Consensus protocols for networks of dynamic agents," American Control Conference, 2003 Proceedings of the 2003, vol. 2, pp. 951-956, June 2003.

[44] Devicenet technical overview, Open Devicenet Vendor Association (ODVA), 2004.

[45] S. D. Pekarek, J. Tichenor, S. D. Sudhoff, J. D. Sauer, D. E. Delisle, and E. J. Zivi, "Overview of a naval combat survivability program," Proceedings of the 13th International Ship Control Systems Symposium, Orlando, Florida, 2003.

[46] S. Peri and N. Mittal, "On termination detection in an asynchronous distributed system." Proceedings of the ISCA International Conference on Parallel and Distributed Computing Systems (PDCS), San Francisco, California, USA, pp. 209-215, Sep 2004.

[47] C. J. Petrie, "Agent-based engineering, the web and intelligence," IEEE Expert, pp. 2429, Dec 1996. 
[48] O. Pfeiffer, A. Ayre, and C. Keydel, Embedded networking with CAN and CANOpen. RTC Books, 2003.

[49] L. Qi and K. Butler-Purry, "Analysis of stability issues during reconfiguration of shipboard power systems," Power Engineering Society General Meeting, 2005. IEEE, no. 485-490, June 2005.

[50] C. Rehtanz, Autonomous systems and intelligent agents in power system control and operation, ser. Power Systems. Springer, 2003.

[51] W. Ren, R. W. Beard, and E. M. Atkins, "A survey of consensus problems in multiagent coordination," American Control Conference, 2005 Proceedings of the 2005, vol. 3, pp. 1859-1864, June 2005.

[52] A. Riera, M. Lama, E. Sanchez, R. Amorim, X. A. Vila, and S. Barro, "Study of communication in a multi-agent system for collaborative learning scenarios," in Parallel, Distributed and Network-Based Processing, 2004., Feb 2004, pp. 233-240.

[53] S9086-KY-STM-010/CH-320R2, Naval Ships Technical Manual Chapter 320, Electric Power Distribution Systems, Direction of Commander, Naval Sea Systems Command, Apr 1998.

[54] G. M. Saunders and J. B. Pollack, "The evolution of communication schemes over continuous channels," Proceedings of the Fourth International Conference on Adaptive Behavior, 1996.

[55] [SC00001L], "FIPA abstract architecture specification," Foundation for Intelligent Physical Agents," Standard, 2002.

[56] [SC00023K], "FIPA agent management specification," Foundation for Intelligent Physical Agents," Standard, 2001. 
[57] [SC00037J], "FIPA communicative act library specification," Foundation for Intelligent Physical Agents," Standard, 2001.

[58] [SC00061G], "FIPA ACL message structure specification," Foundation for Intelligent Physical Agents," Standard, 2001.

[59] [SC00067F], "FIPA agent message transport service specification," Foundation for Intelligent Physical Agents," Standard, 2001.

[60] [SC00069G], "FIPA ACL message representation in bit-efficient encoding specification," Foundation for Intelligent Physical Agents," Standard, 2001.

[61] [SC00088D], "FIPA ACL message transport envelope representation in bit-efficient encoding specification," Foundation for Intelligent Physical Agents," Standard, 2001.

[62] [SC00091E], "FIPA device ontology specification," Foundation for Intelligent Physical Agents," Standard, 2001.

[63] G. Schrott, "A multi-agent distributed real time system for a microprocessor fieldbus network," Real-Time Systems, 1995. Proceedings., Seventh Euromicro Workshop on, pp. 302-307, June 1995.

[64] R. G. Smith, "The contractnet protocol: High-level communication and control in a distributed problem solver," IEEE transactions on Computers, vol. C-29, no. 12, Dec 1980.

[65] J. Solanki and N. Schulz, "Using intelligent multiagent systems for shipboard power systems reconfiguration," Intelligent Systems Application to Power Systems, 2005. Proceedings of the 13th International Conference on, Nov 2005.

[66] B. K. Song, J. W. Jung, Y. H. Lee, and S. S. An, "MMTP - Multicast and Multidestination Transport Protocol," Communications, 1994. ICC 94, SUPERCOMM/ICC '94, vol. 3, pp. 1331-1335, May 1994. 
[67] S. Srivastava and K. Butler-Purry, "A probability based predictive reconfiguration method for shipboard power systems," Probabilistic Methods Applied to Power Systems, 2004 International Conference on, pp. 813-818, Sept 2004.

[68] D. M. Staszesky, "Use of virtual agents to effect intelligent distribution automation," Power Engineering Society General Meeting, 2006. IEEE, June 2006.

[69] L. Steels, "Self organizing vocabularies," in Artificial Life, C. G. Langton and K. Shimohara, Eds., Nara Japan, 1996, pp. 179-184.

[70] Sudhoff, Gllover, Zak, Pekarek, Zivi, Delisle, and Clayton, "Stability analysis methodologies for DC power distribution systems," Proceedings of the 13th International Ship Control Systems Symposium, 2003, orlando, Florida.

[71] K. P. Sycara, "Multiagent systems," American Association for Artificial Intelligence, pp. 79-92, Summer 1998.

[72] G. Tel, Introduction to Distributed Algorithms. Cambridge University Press, 1994.

[73] P. Urlings, J. Tweedale, C. Sioutis, and N. Ichalkaranje, Intelligent Agents and situation awareness, ser. Lecture Notes in Computer Science. Springer Berlin / Heidelberg, 2003, vol. $2774 / 2003$, pp. $723-733$.

[74] G. Weiss, Ed., Multiagent Systems. $\quad$ MIT press, 1999.

[75] M. Wooldridge, "Intelligent agents: the key concepts," Lecture notes in Computer Science, Springer Berlin Heidelberg, vol. 2322/2002, p. 3, 2002, multi-Agent-Systems and Applications II : 9th ECCAI-ACAI/EASSS 2001, AEMAS 2001, HoloMAS 2001. Selected Revised Papers.

[76] M. Wooldridge and N. R. Jennings, "Intelligent agents: Theory and practice," The Knowledge Sharing Review, vol. 10:2, pp. 115-152, 1995. 
[77] Z. Zhang, J. McCalley, V. Vishwanathan, and V. Honavar, "Multiagent system solutions for distributed computing, communications, and data integration needs in the power

industry," Power Engineering Society General Meeting, 2004. IEEE, pp. 45-49, June 2004 . 